Supporting Information

\title{
Theoretical Insight into Nonadiabatic Proton-Coupled Electron Transfer Mechanism of Reduced Flavin Oxygenation
}

\author{
Yanling Luo, Ya-Jun Liu*
}

Key Laboratory of Theoretical and Computational Photochemistry, Ministry of Education, College of Chemistry, Beijing Normal University, Beijing 100875, China

\section{Contents}

Additional Figures and Tables ................................................................................

Absolute energies and available imaginary frequencies of all optimized structures.... 18

The XYZ Cartesian Coordinates of Key Structures Reported (unit: Ångstrom)..........20 


\section{List of Figures}

Figure S1. The redox and acid-base equilibria of flavins. 4

Figure S2. The comparison of the optimized bond lengths for the singlet reactant $\mathrm{R}_{\mathrm{S}}$ :

${ }^{1}\left(\mathrm{LF}^{-}\right.$red... $\left.\mathrm{O}_{2}\right)$ by the four different sets of DFT methods in the gas phase, which are the (U)wB97XD/6-311G**，(U)CAM-B3LYP/6-311G**，(U)CAM-B3LYP/6-311+G** and

(U)CAM-B3LYP/6-311++G** .5

Figure S3. The comparison of the optimized bond lengths for the singlet transition state $\mathrm{TS}_{\mathrm{S}}$ by the four different sets of DFT methods in the gas phase, which are the (U)wB97XD/6-311G**, (U)CAM-B3LYP/6-311G**, (U)CAM-B3LYP/6-311+G** and (U)CAM-B3LYP/6-311++G**. 6

Figure S4. The comparison of the optimized bond lengths for the singlet product $\mathrm{P}_{\mathrm{S}}{ }^{1} \mathrm{LFOO}_{\mathrm{H}}$ by the four different sets of DFT methods in the gas phase, which are the (U)wB97XD/6-311G**, (U)CAM-B3LYP/6-311G**, (U)CAM-B3LYP/6-311+G** and (U)CAM-B3LYP/6-311++G** 7

Figure S5. The active space orbitals used in MS-CASPT2//CAM-B3LYP calculations to investigate the adiabatic PECs for reaction between oxygen and reduced LF anion. 9

Figure S6. The active space orbitals used in MS-CASPT2//CASSCF calculations to optimized the ISC point. 9

Figure S7. The relative energies of the reactants and products in different states $\left(\mathrm{S}_{0}\right.$ and $\left.\mathrm{T}_{1}\right)$ and different environments (the gas phase, benzene, water and DMSO solvents) computed at (U)CAM-B3LYP/6-311G** level.

Figure S8. The structures of the key optimized reactants and products computed at (U)CAM-B3LYP/6-311G** level. 10

Figure S9. The $\mathrm{S}_{0}$ and $\mathrm{T}_{1}$ PECs of the reaction between oxygen and reduced LF anion in the gas phase calculated at the UCAM-B3LYP/6-311G** level. 11

Figure S10. The description of singlet reaction pathway between oxygen and reduced LF anion in benzene. The PEC (a), the key structures (b), the NPA charge of $\mathrm{O}_{2}$ moiety (c) and the geometric parameters (d) were computed at the UCAM-B3LYP/6-311G** level. .12

Figure $\mathrm{S} 11$. The $\mathrm{S}_{0}$ and $\mathrm{T}_{1}$ PECs of the reaction between oxygen and reduced LF anion in benzene calculated at the UCAM-B3LYP/6-311G** level.

Figure S12. The description of singlet reaction pathway between oxygen and reduced LF anion 
in DMSO. The PEC (a), the key structures (b), the NPA charge of $\mathrm{O}_{2}$ moiety (c) and the geometric parameters $(\mathrm{d})$ were computed at the UCAM-B3LYP/6-311G** level.

Figure $\mathrm{S} 13$. The $\mathrm{S}_{0}$ and $\mathrm{T}_{1}$ PECs of the reaction between oxygen and reduced LF anion in DMSO calculated at the UCAM-B3LYP/6-311G** level. .15

Figure S14. The description of singlet reaction pathway between oxygen and reduced LF anion in water. The PEC (a), the key structures (b), the NPA charge of $\mathrm{O}_{2}$ moiety (c) and the geometric parameters $(\mathrm{d})$ were computed at the UCAM-B3LYP/6-311G** level. 16

Figure $\mathrm{S} 15$. The $\mathrm{S}_{0}$ and $\mathrm{T}_{1}$ PECs of the reaction between oxygen and reduced LF anion in water calculated at the UCAM-B3LYP/6-311G** level. 17

Figure S16. The Mulliken charge of $\mathrm{O}_{2}$ moiety in the reaction path calculated by MS-CASPT2//CAM-B3LYP method

\section{List of Tables}

Table $\mathrm{S} 1$. The predicted free energies $(\Delta G \mathrm{in} \mathrm{kcal} / \mathrm{mol})$ for the singlet reactant $\mathrm{R}_{\mathrm{S}}:^{1}\left(\mathrm{LF}^{-}{ }^{-}{ }{ } \ldots \mathrm{O}_{2}\right)$, transition state $\mathrm{TS}_{\mathrm{S}}$ and product $\mathrm{P}_{\mathrm{S}}{ }^{1} \mathrm{LFOO}_{\mathrm{H}}{ }^{-}$by the four different sets of DFT methods in the gas phase. 8

Table S2. The predicted reaction energy barriers $(\Delta G$ in $\mathrm{kcal} / \mathrm{mol})$ and minimum NPA charge of $\mathrm{O}_{2}$ moiety $\left(\rho \mathrm{O}_{2}\right.$ in $e$ ) during the singlet reaction path in different environments. 11

Table S3. The MS-CASPT2//SA6-CAS(12,9)/ANO-RCC-VDZP calculated weightings of the electronic configurations (weight $\geqslant 0.1$ ) at the ISC point in the $\mathrm{S}_{0}$ and $\mathrm{T}_{1}$ states. 18

Table S4. Absolute energies (in Hartree) and available imaginary frequencies (in $\mathrm{cm}^{-1}$ ) of optimized structures calculated at (U)CAM-B3LYP/6-311G** level. 18

Table S5. Absolute energies (in Hartree) and available imaginary frequencies (in $\mathrm{cm}^{-1}$ ) of optimized structures calculated by four different sets of DFT methods in the gas phase....19 Table S6. Absolute energies (in Hartree) of the optimized ISC point in the $\mathrm{S}_{0}$ state and $\mathrm{T}_{1}$ states calculated at CASSCF and MS-CASPT2 level respectively. 20 


\section{Additional Figures and Tables}
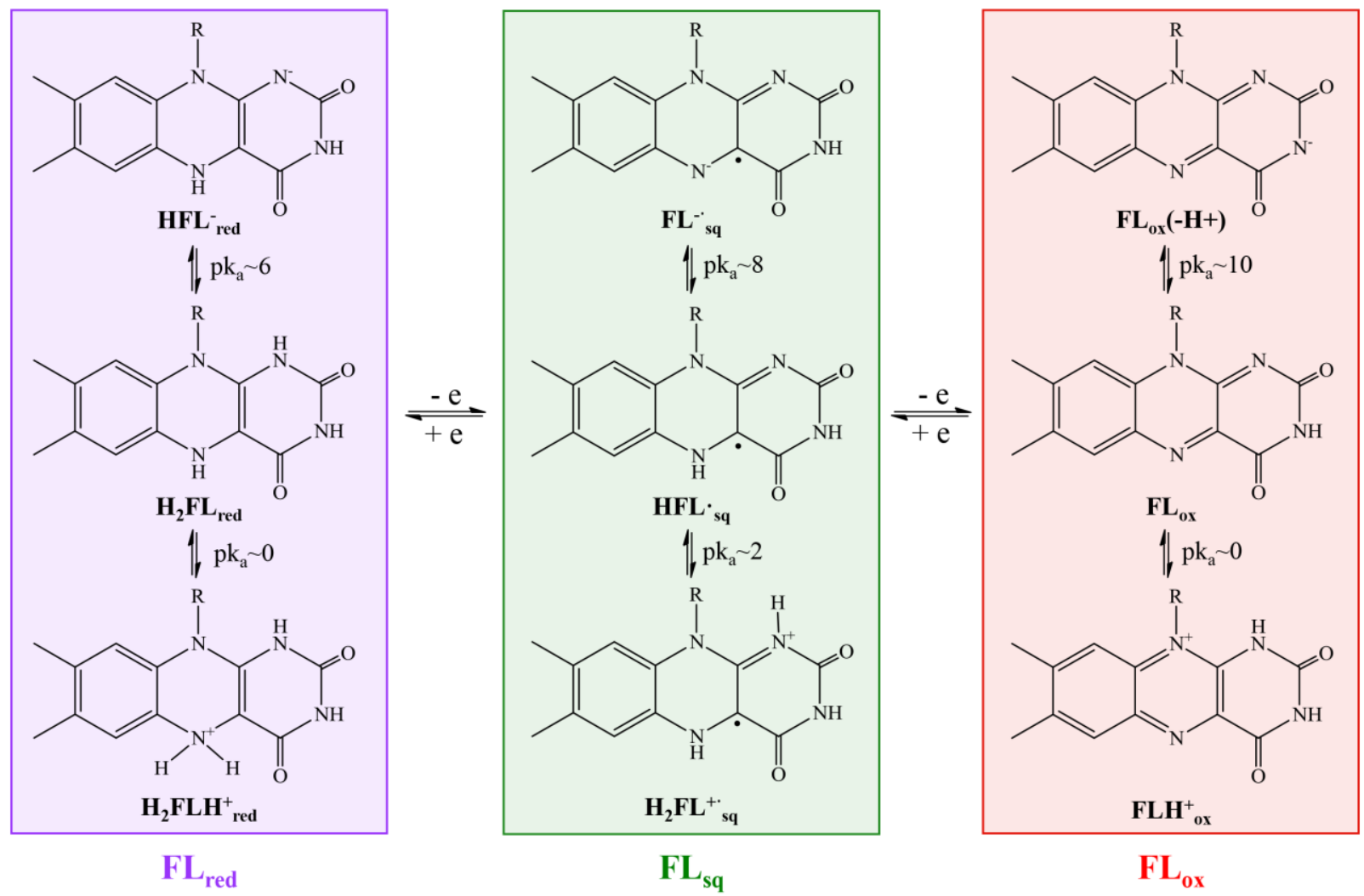

Figure S1. The redox and acid-base equilibria of flavins. 

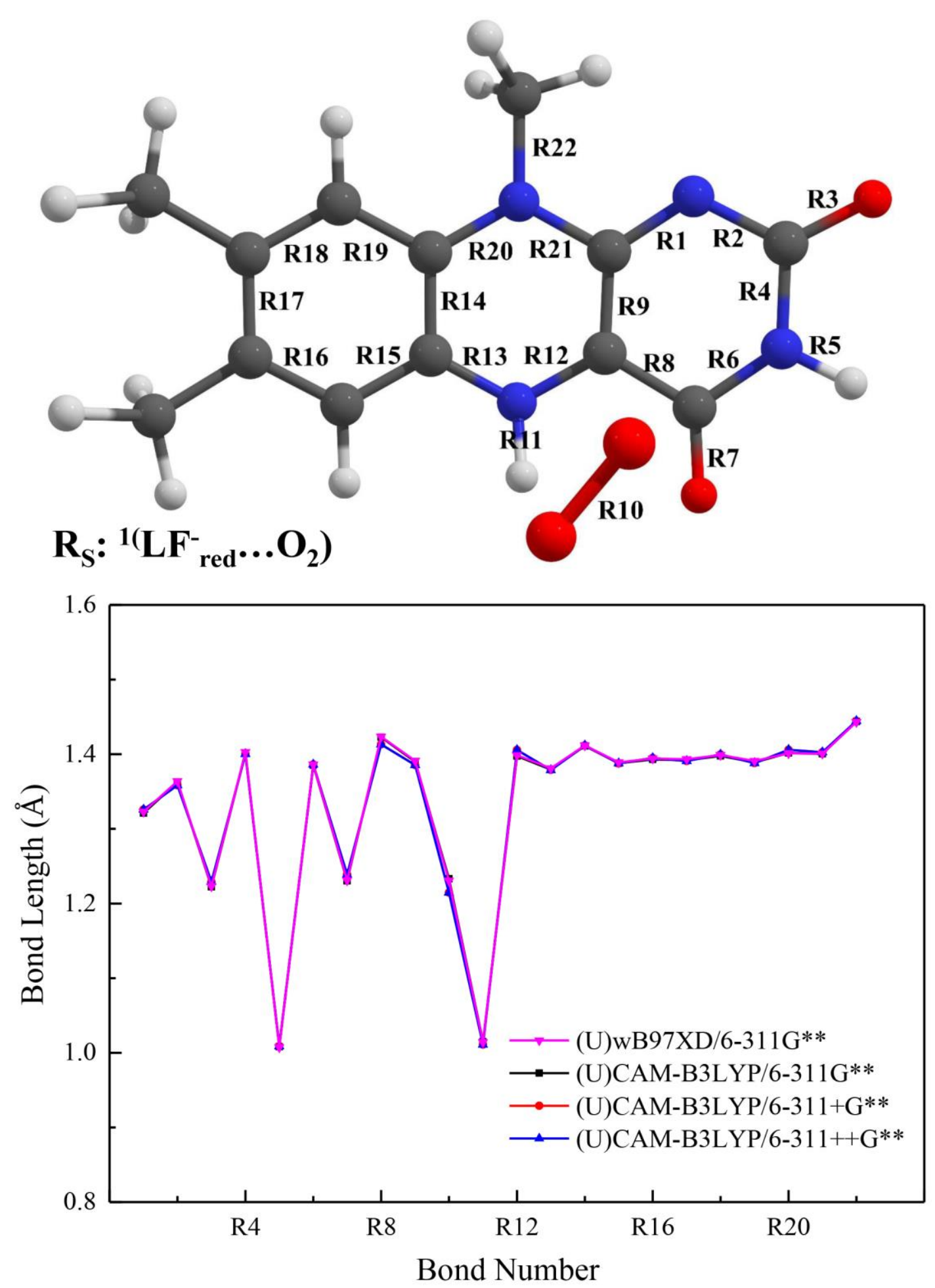

Figure S2. The comparison of the optimized bond lengths for the singlet reactant $\mathrm{R}_{\mathrm{S}} \mathrm{I}^{1}\left(\mathrm{LF}^{-}{ }_{\text {red }} \ldots \mathrm{O}_{2}\right)$ by the four different sets of DFT methods in the gas phase, which are the (U)wB97XD/6-311G**, (U)CAM-B3LYP/6-311G**, (U)CAM-B3LYP/6-311+G** and (U)CAM-B3LYP/6-311++G**. 

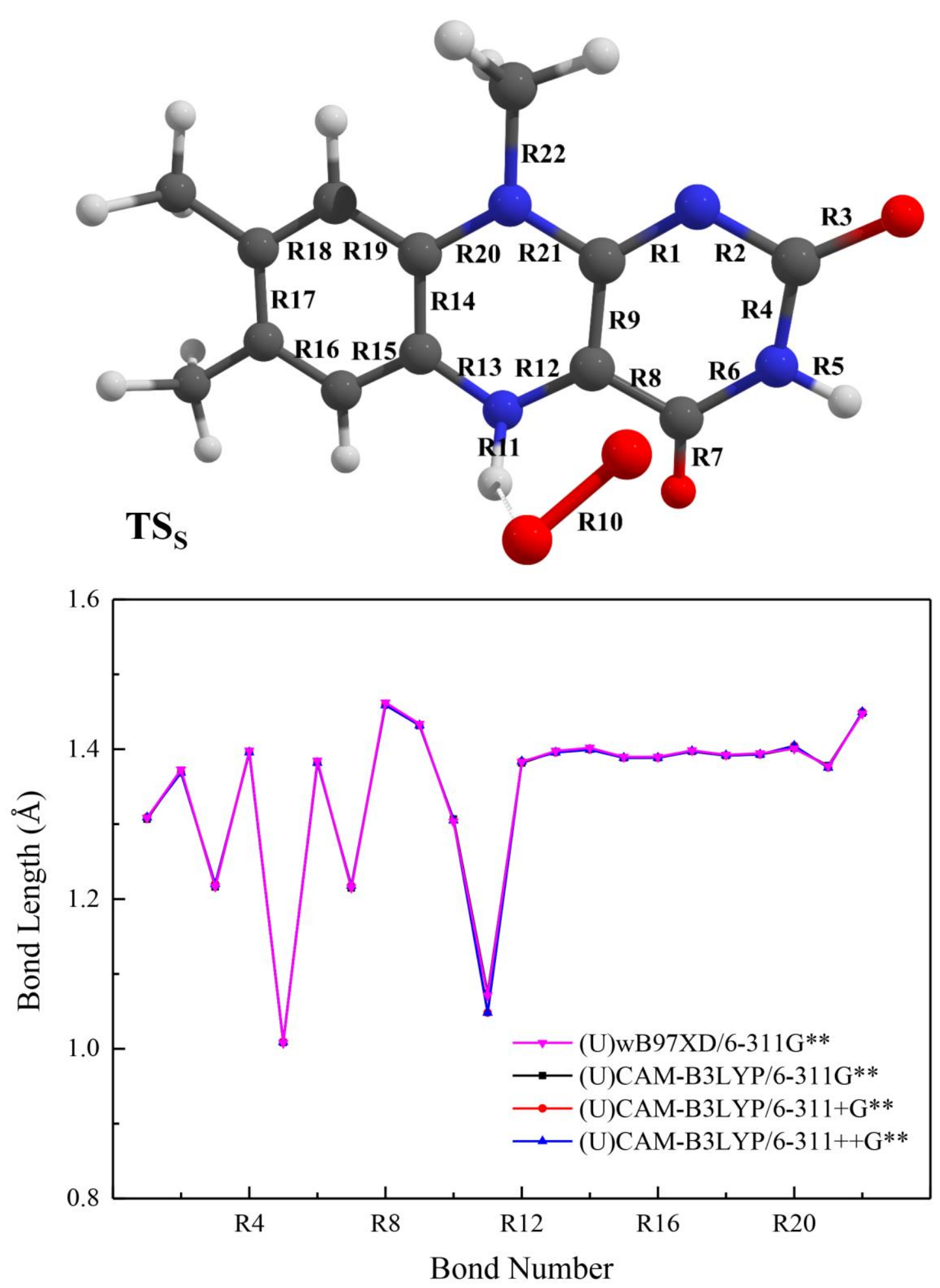

Figure S3. The comparison of the optimized bond lengths for the singlet transition state $\mathrm{TS}_{\mathrm{S}}$ by the four different sets of DFT methods in the gas phase, which are the (U)wB97XD/6-311G**, (U)CAM-B3LYP/6-311G**, (U)CAM-B3LYP/6-311+G** and (U)CAM-B3LYP/6-311++G**. 

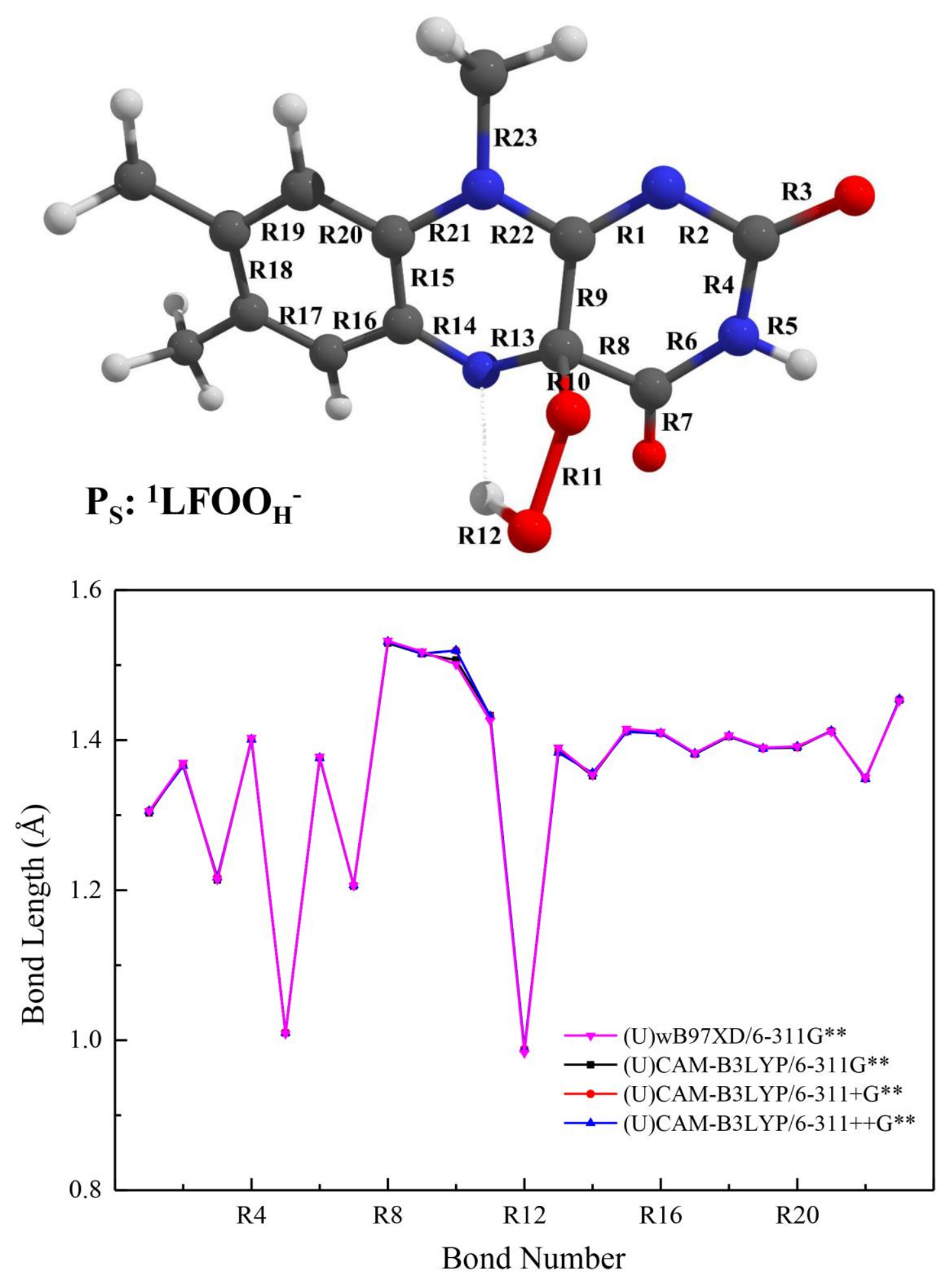

Figure S4. The comparison of the optimized bond lengths for the singlet product $\mathrm{Ps}_{\mathrm{S}}{ }^{1} \mathrm{LFOO}_{\mathrm{H}}-$ by the four different sets of DFT methods in the gas phase, which are the (U)wB97XD/6-311 $\mathrm{G}^{* *}$, (U)CAM-B3LYP/6-311G**, (U)CAM-B3LYP/6-311+G** and (U)CAM-B3LYP/6-311++G**. 
Table S1. The predicted free energies $(\Delta G$ in $\mathrm{kcal} / \mathrm{mol})$ for the singlet reactant $\mathrm{R}_{\mathrm{S}}:{ }^{1}\left(\mathrm{LF}^{-}{ }_{\text {red... }} \mathrm{O}_{2}\right)$, transition state $\mathrm{TS}_{\mathrm{S}}$ and product $\mathrm{P}_{\mathrm{S}}:{ }^{1} \mathrm{LFOO}_{\mathrm{H}}{ }^{-}$by the four different sets of DFT methods in the gas phase.

\begin{tabular}{|c|c|c|c|c|}
\hline & $\begin{array}{c}\text { (U)CAM-B3LYP/6-311G } \\
* *\end{array}$ & $\begin{array}{c}\text { (U)CAM-B3LYP/6-311+G } \\
* *\end{array}$ & $\begin{array}{c}\text { (U)CAM-B3LYP/6-311++G } \\
* *\end{array}$ & $\begin{array}{c}\text { (U)wB97X } \\
\mathrm{D} \\
/ 6-311 \mathrm{G}^{* * *}\end{array}$ \\
\hline $\begin{array}{c}{ }^{1}\left(\mathrm{LF}_{\text {red }}^{-}\right. \\
\left.\cdots \mathrm{O}_{2}\right)\end{array}$ & 0 & 0 & 0 & 0 \\
\hline $\mathrm{TS}_{\mathrm{S}}$ & 10.13 & 9.10 & 9.07 & 9.86 \\
\hline${ }^{1} \mathrm{LFOO}_{\mathrm{H}}{ }^{-}$ & -8.78 & -8.81 & -8.90 & -9.90 \\
\hline
\end{tabular}

Active Space: The singlet reaction path was first obtained using the DFT method (UCAM-B3LYP/6-311G** with broken-symmetry technology). Then the MS-CASPT2 method was employed to calculate the energies at the DFT calculated reaction path. In order to describe the PECT reaction process, the $(12,9)$ active space was chosen, which includes the $\pi$ and $\pi^{*}$ orbitals of $\mathrm{C}_{4 \mathrm{a}}-\mathrm{C}_{10 \mathrm{a}}$ bond, $\sigma$ and $\sigma^{*}$ orbitals of $\mathrm{N}_{5}-\mathrm{H}$ bond, two degenerated $\pi^{*}$ orbitals of $\mathrm{O}_{2}$, and $\mathrm{n}$ orbital of $\mathrm{N}_{1}$ (Figure S5). These orbitals are valuable correlated active orbitals which involve the reaction in bonding and bond breaking.

For the ISC optimization, a new $(12,9)$ active space was selected to describe the ${ }^{3}\left({ }^{2} \Pi_{\mathrm{g}}-\mathrm{O}_{2}+\mathrm{LF}^{-}\right) /$closed-shell intersection better, which $\sigma$ and $\sigma^{*}$ orbitals of $\mathrm{N}_{5}-\mathrm{H}$ bond was replaced by $\pi$ and $\pi^{*}$ orbitals of $\mathrm{C}=\mathrm{O}$ (Figure $\mathrm{S} 6$ ). 

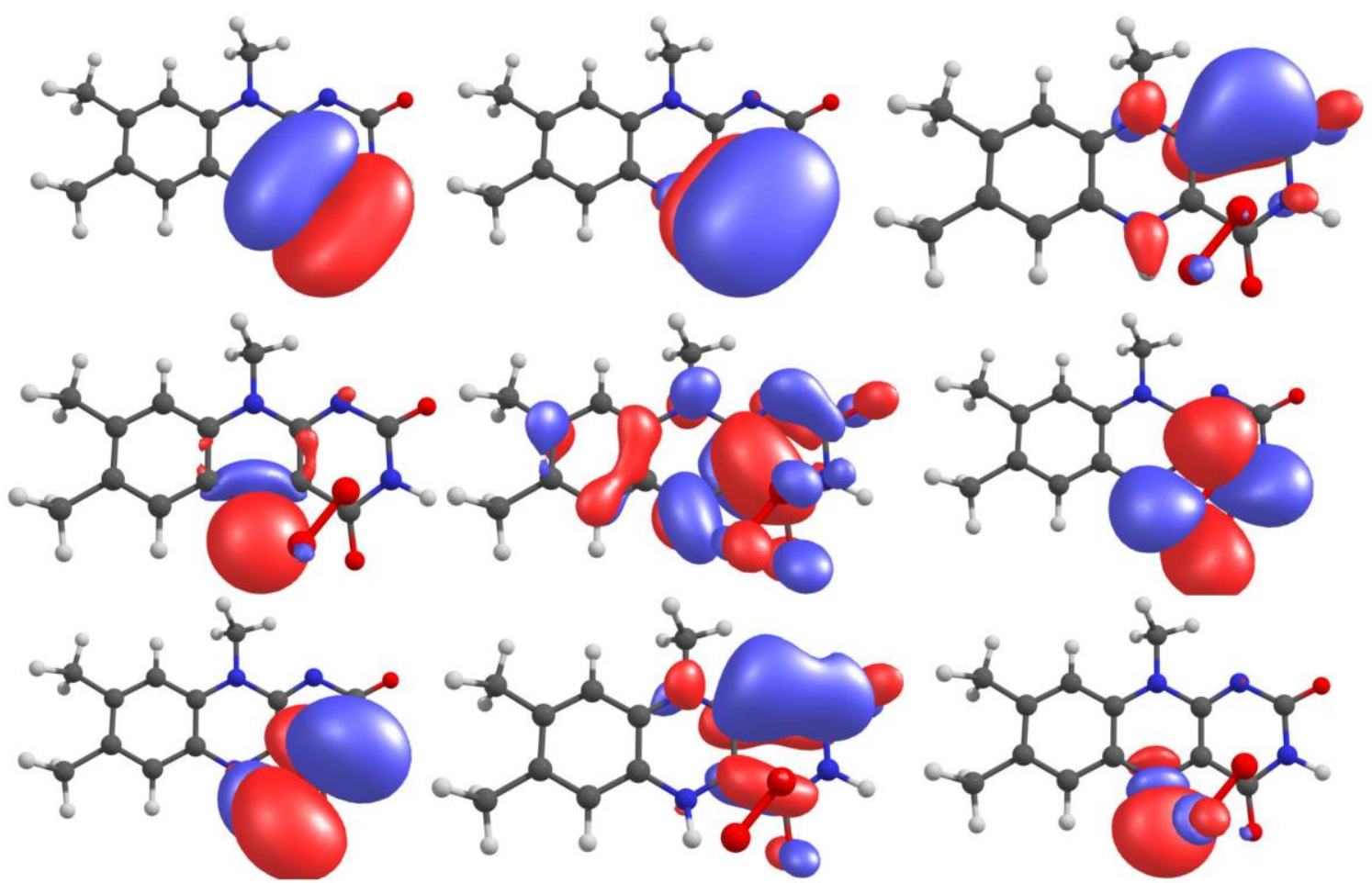

Figure S5. The active space orbitals used in MS-CASPT2//CAM-B3LYP calculations to investigate the adiabatic PECs for reaction between oxygen and reduced LF anion.
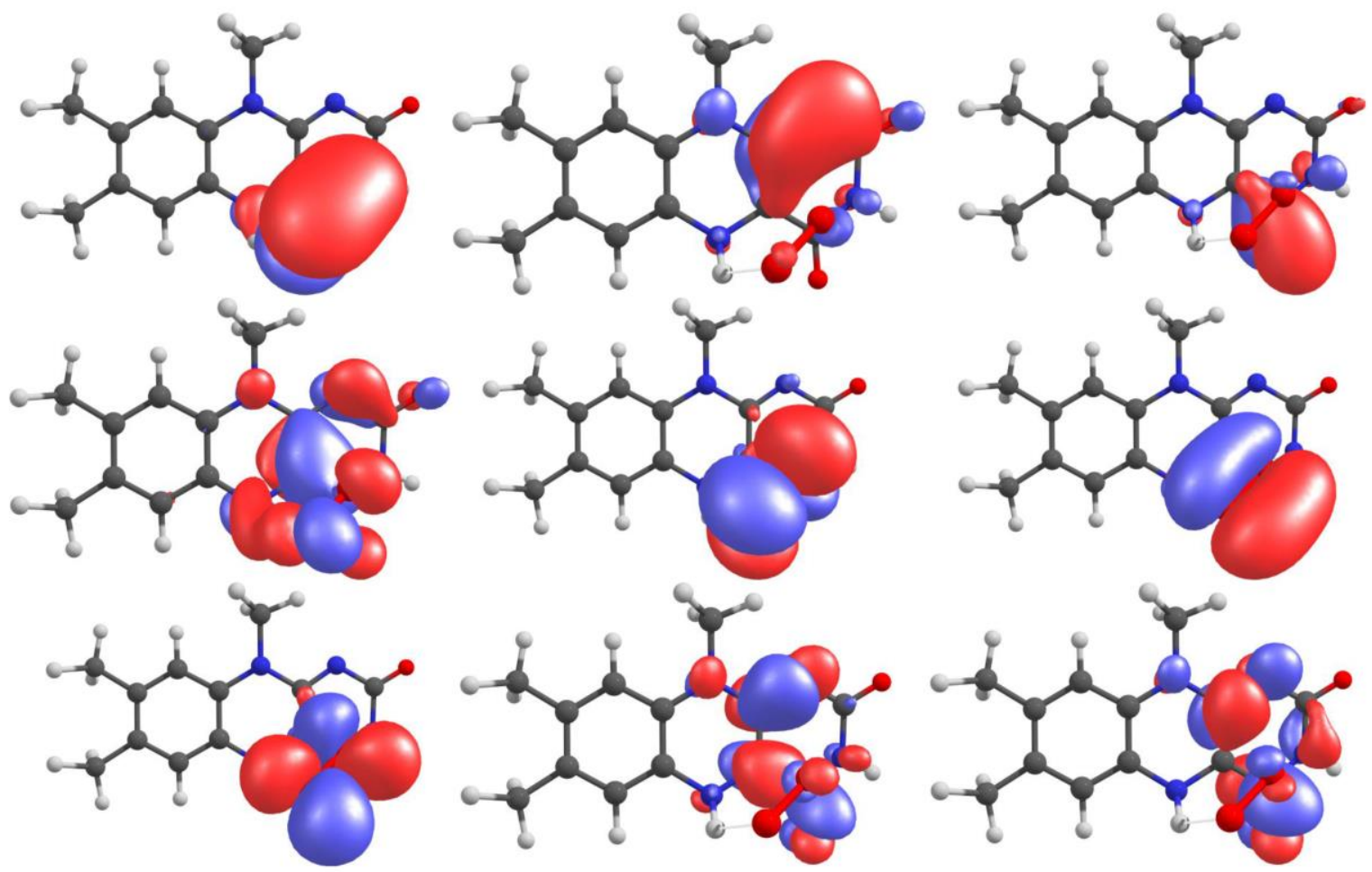

Figure S6. The active space orbitals used in MS-CASPT2//CASSCF calculations to optimized the ISC point. 


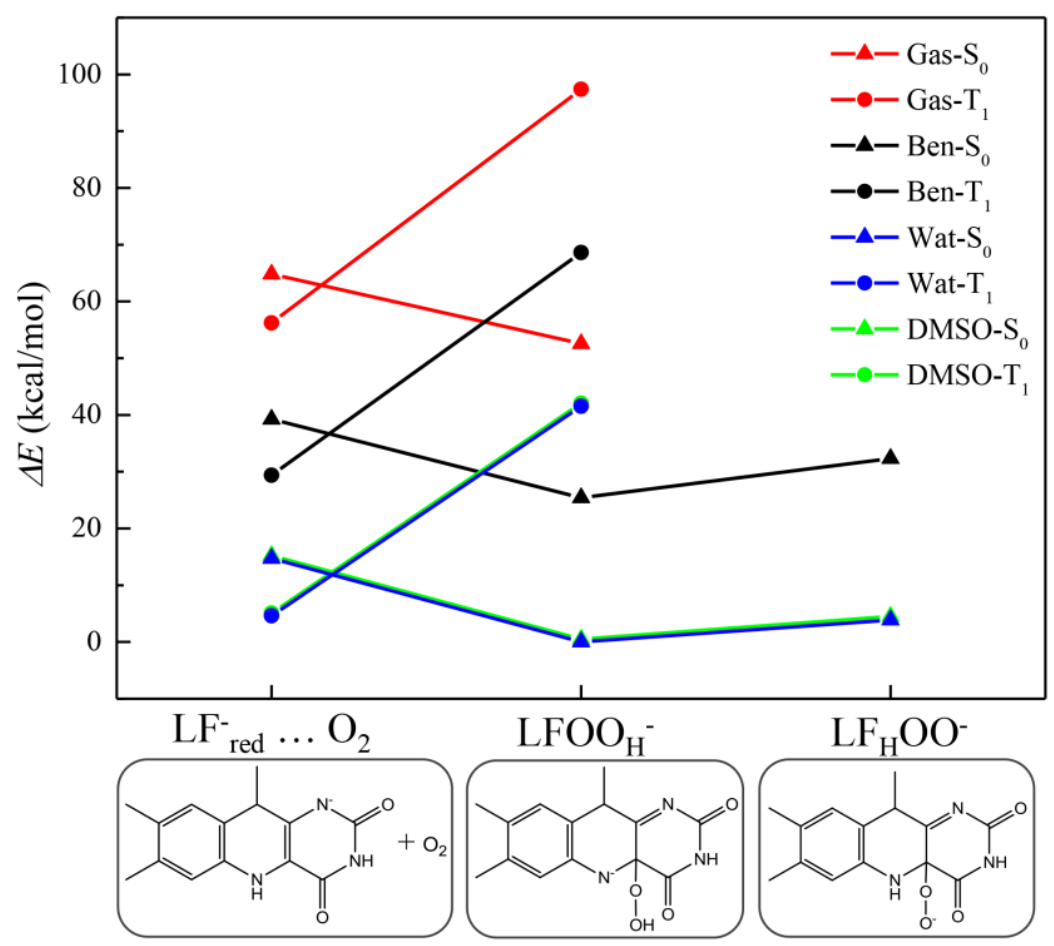

Figure S7. The relative energies of the reactants and products in different states $\left(\mathrm{S}_{0}\right.$ and $\left.\mathrm{T}_{1}\right)$ and different environments (the gas phase, benzene, water and DMSO solvents) computed at (U)CAM-B3LYP/6-311G** level.


Figure S8. The structures of the key optimized reactants and products computed at (U)CAM-B3LYP/6-311G** level. 


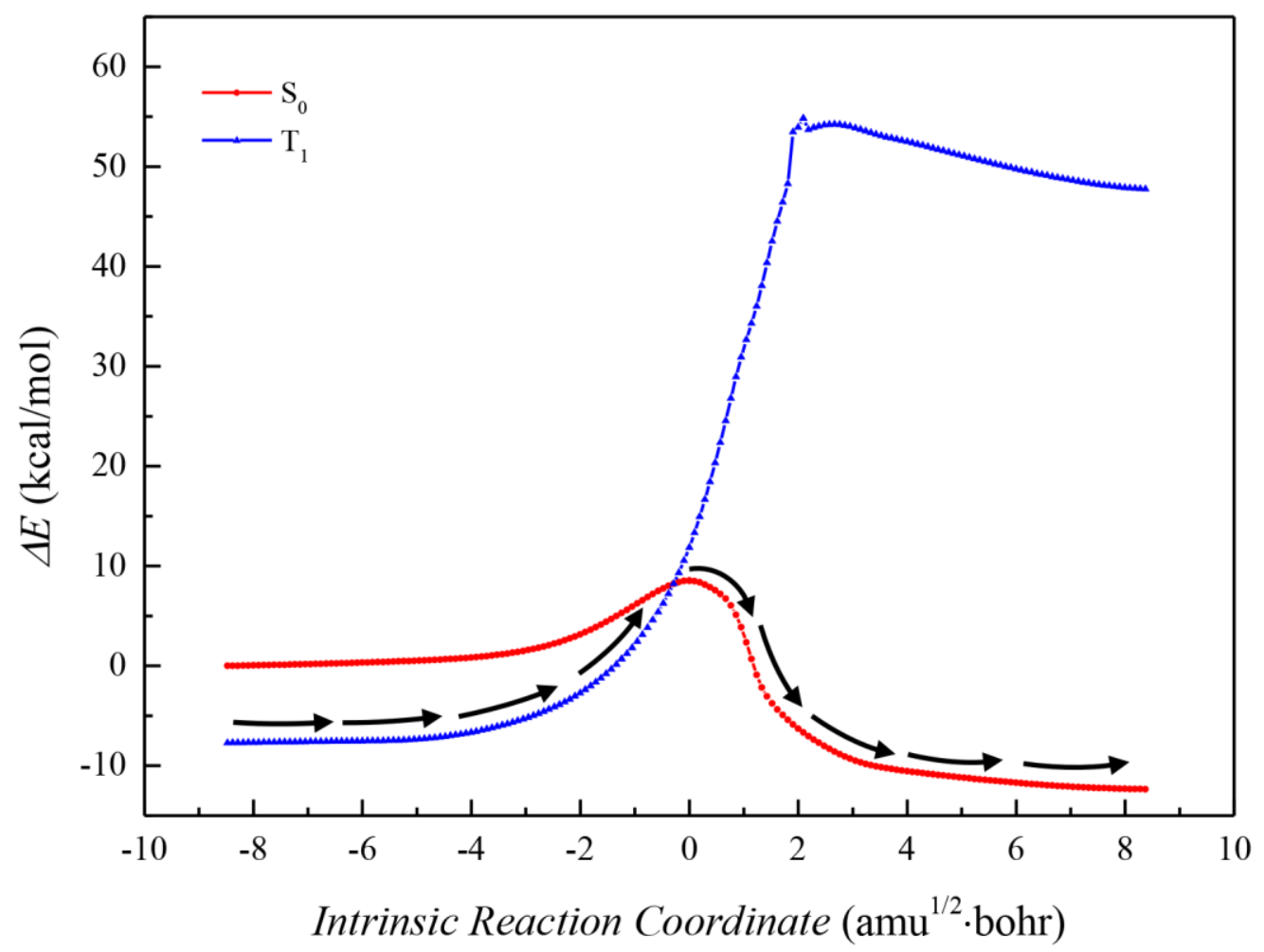

Figure $\mathrm{S} 9$. The $\mathrm{S}_{0}$ and $\mathrm{T}_{1}$ PECs of the reaction between oxygen and reduced LF anion in the gas phase calculated at the UCAM-B3LYP/6-311G** level.

Table S2. The predicted reaction energy barriers $(\Delta G$ in $\mathrm{kcal} / \mathrm{mol})$ and minimum NPA charge of $\mathrm{O}_{2}$ moiety $\left(\mathrm{\rho O}_{2}\right.$ in $e$ ) during the singlet reaction path in different environments.

\begin{tabular}{ccccc} 
& Gas & Benzene & DMSO & Water \\
\hline$\Delta G$ & 10.13 & 6.90 & 5.75 & 5.71 \\
$\rho_{O 2}$ & -0.89 & -0.94 & -0.99 & -0.99 \\
\hline
\end{tabular}


(a)

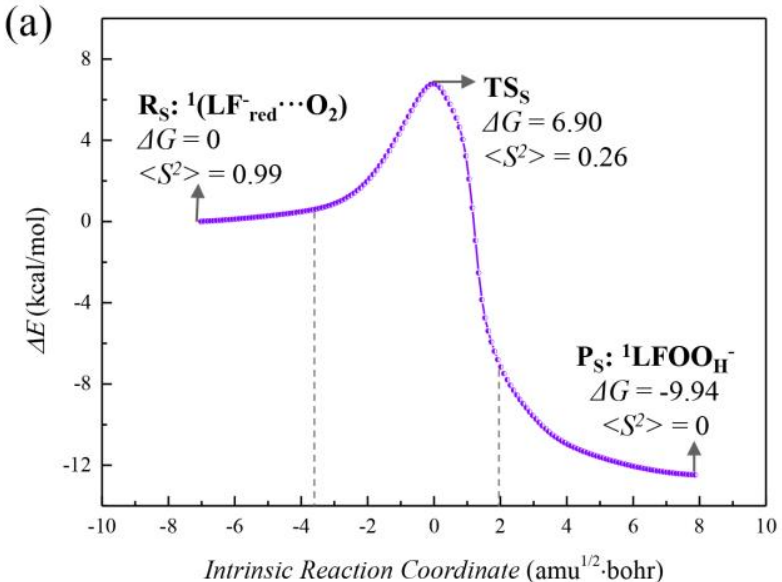

(b)
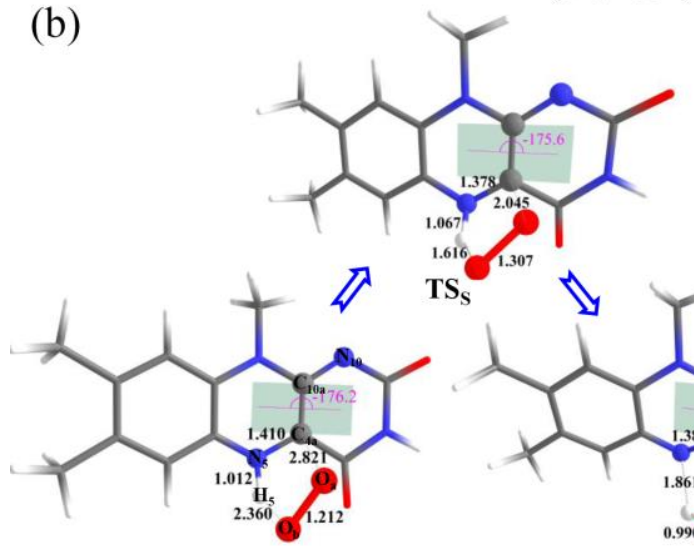

$\mathbf{R}_{\mathrm{S}}:{ }^{1}\left(\mathbf{L F} \cdots \mathbf{O}_{2}\right)$

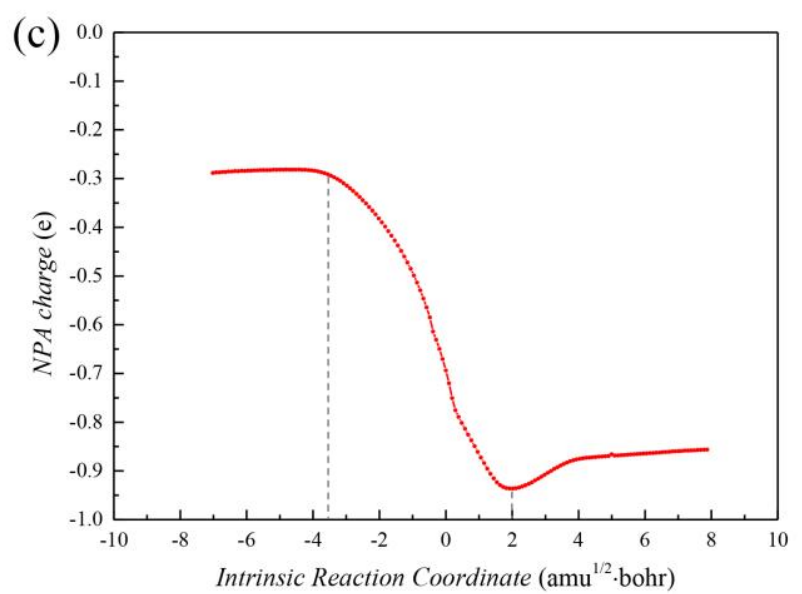

(d)

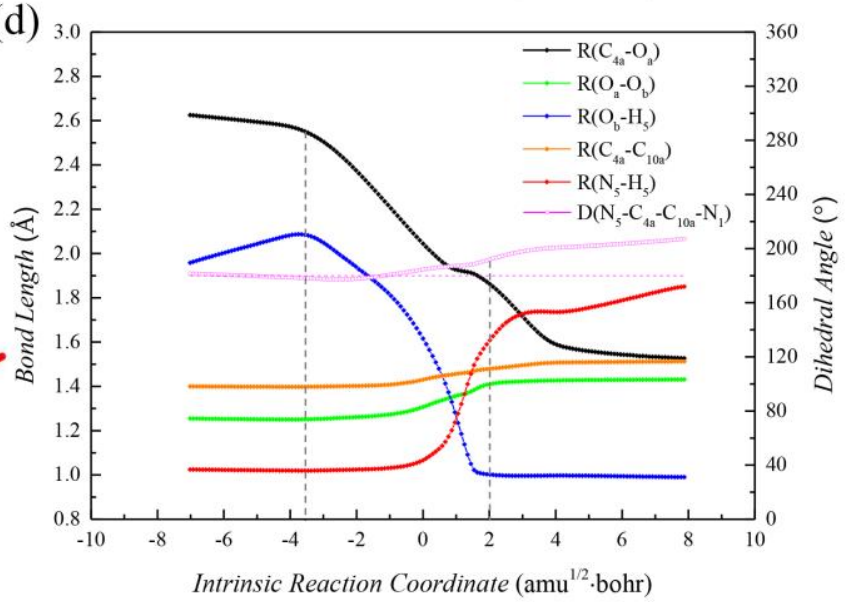

Figure S10. The description of singlet reaction pathway between oxygen and reduced LF anion in benzene. The PEC (a), the key structures (b), the NPA charge of $\mathrm{O}_{2}$ moiety (c) and the geometric parameters (d) were computed at the UCAM-B3LYP/6-311G** level. The key atoms $\left(\mathrm{N}_{1}, \mathrm{C}_{10 \mathrm{a}}, \mathrm{C}_{4 a}\right.$, $\mathrm{N}_{5}, \mathrm{H}_{5}, \mathrm{O}_{\mathrm{a}}$ and $\left.\mathrm{O}_{b}\right)$ are highlighted in black, and the dihedral angle $\left(\mathrm{N}_{5}-\mathrm{C}_{4 a}-\mathrm{C}_{10 \mathrm{a}}-\mathrm{N}_{1}\right)$ in fuchsia. Here and hereunder, gray, blue, white, and red balls represent carbon, nitrogen, hydrogen, and oxygen atoms, respectively. The units are angstrom $(\AA)$ for bond distance, degrees for the dihedral angle, and $\mathrm{kcal} / \mathrm{mol}$ for Gibbs energy change $(\Delta G)$. 


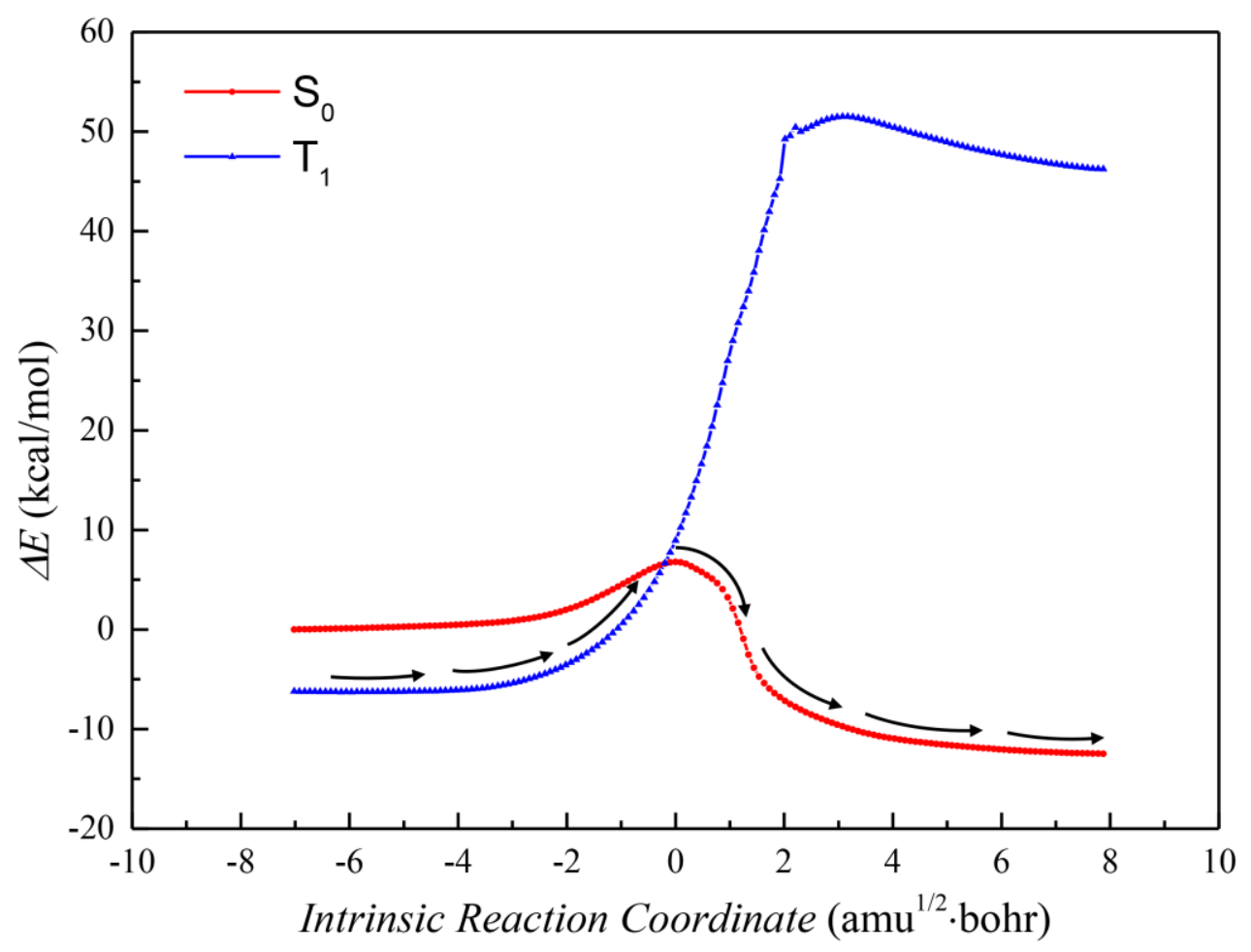

Figure $\mathrm{S} 11$. The $\mathrm{S}_{0}$ and $\mathrm{T}_{1}$ PECs of the reaction between oxygen and reduced LF anion in benzene calculated at the UCAM-B3LYP/6-311G** level. 


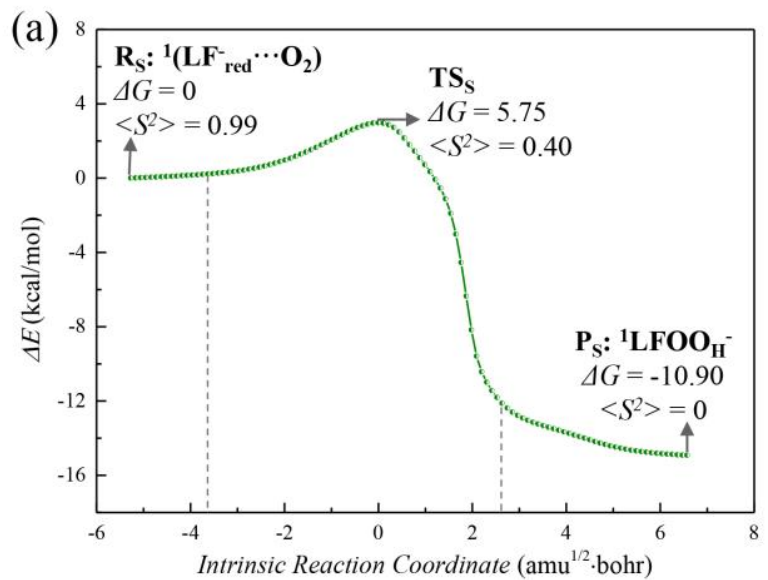

(b)
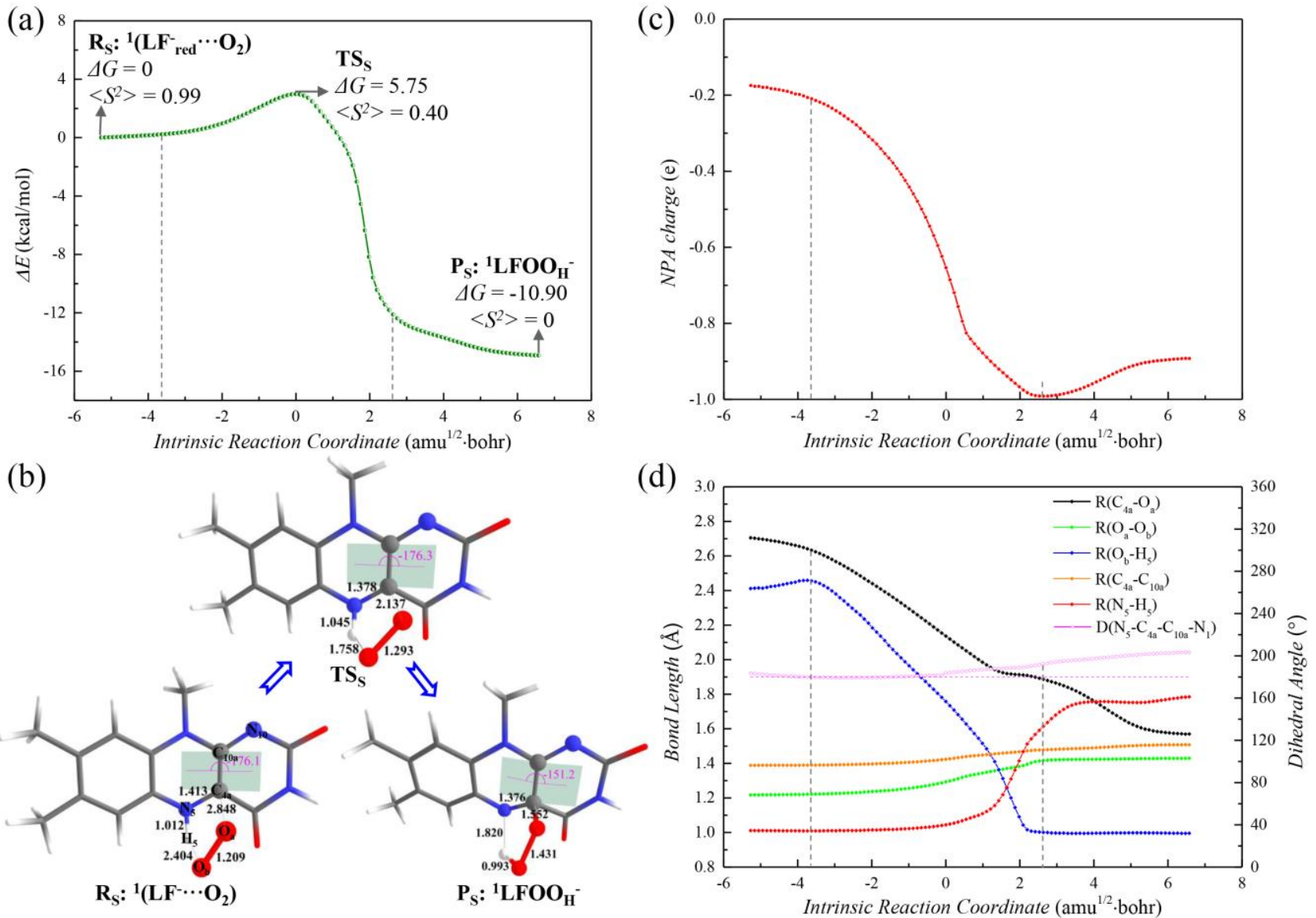

Figure S12. The description of singlet reaction pathway between oxygen and reduced LF anion in DMSO. The PEC (a), the key structures (b), the NPA charge of $\mathrm{O}_{2}$ moiety (c) and the geometric parameters (d) were computed at the UCAM-B3LYP/6-311G** level. 




Figure S13. The $\mathrm{S}_{0}$ and $\mathrm{T}_{1}$ PECs of the reaction between oxygen and reduced LF anion in DMSO calculated at the UCAM-B3LYP/6-311G** level. 

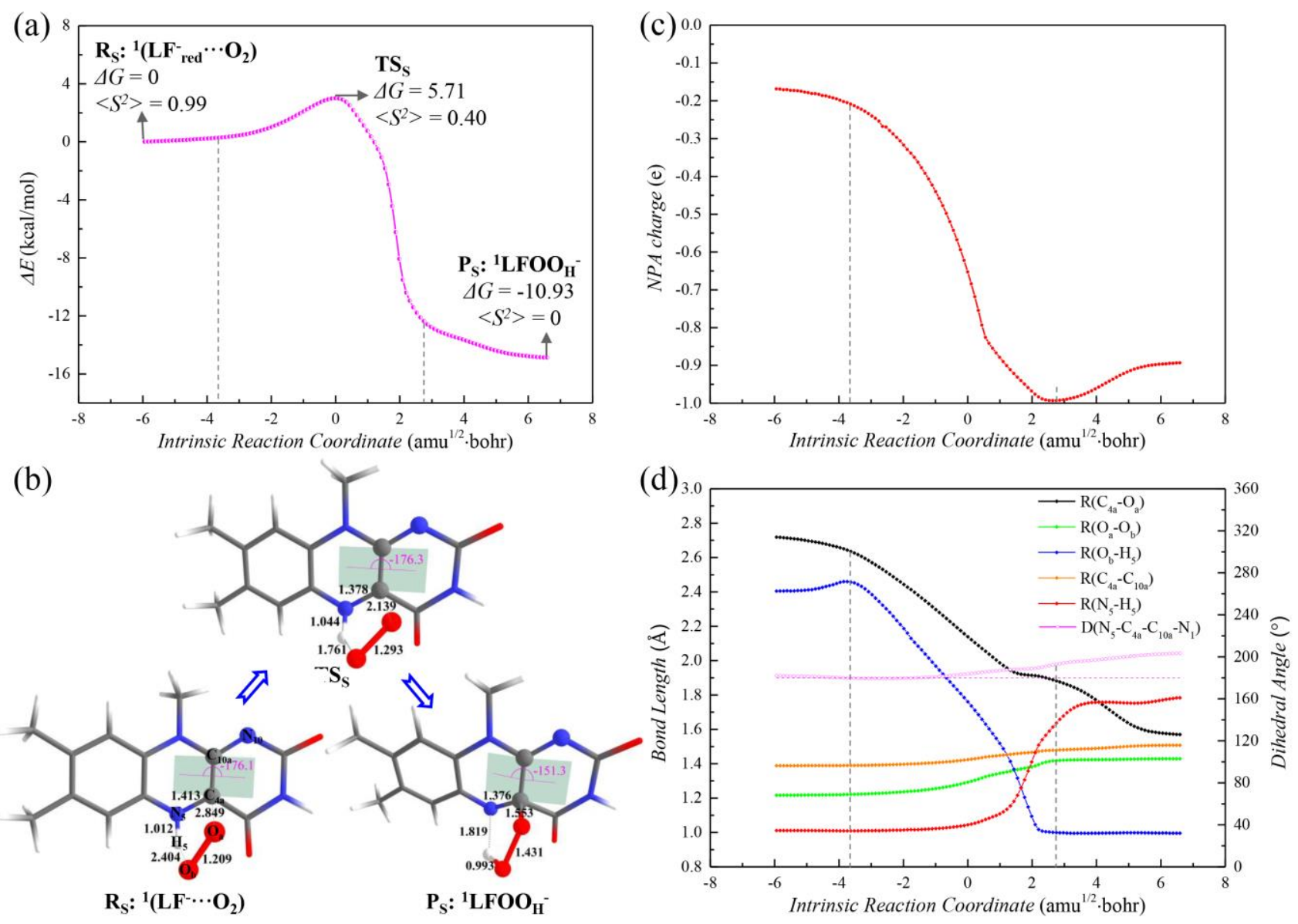

Figure S14. The description of singlet reaction pathway between oxygen and reduced LF anion in water. The PEC (a), the key structures (b), the NPA charge of $\mathrm{O}_{2}$ moiety (c) and the geometric parameters (d) were computed at the UCAM-B3LYP/6-311G** level. 


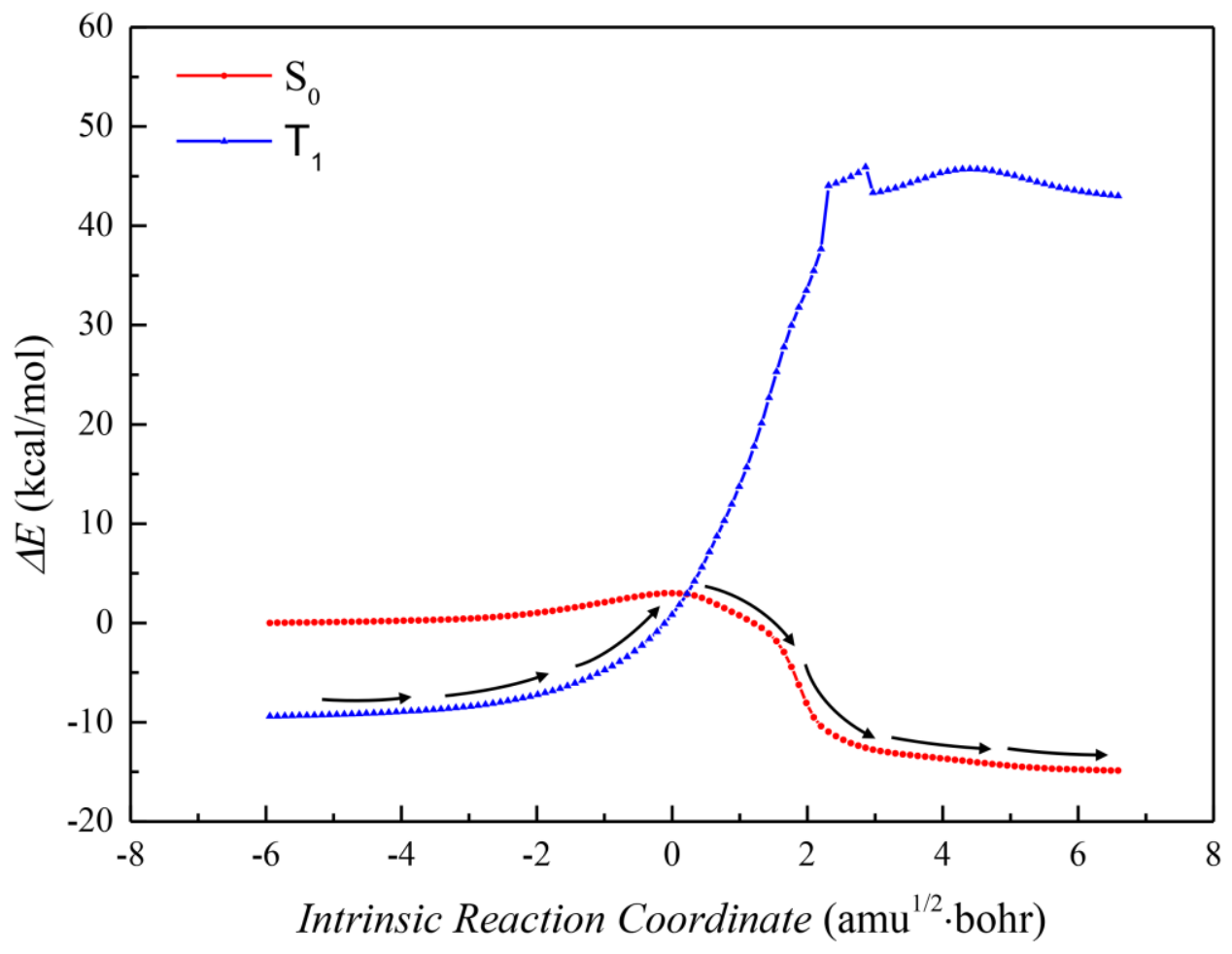

Figure $\mathrm{S} 15$. The $\mathrm{S}_{0}$ and $\mathrm{T}_{1}$ PECs of the reaction between oxygen and reduced LF anion in water calculated at the UCAM-B3LYP/6-311G** level.

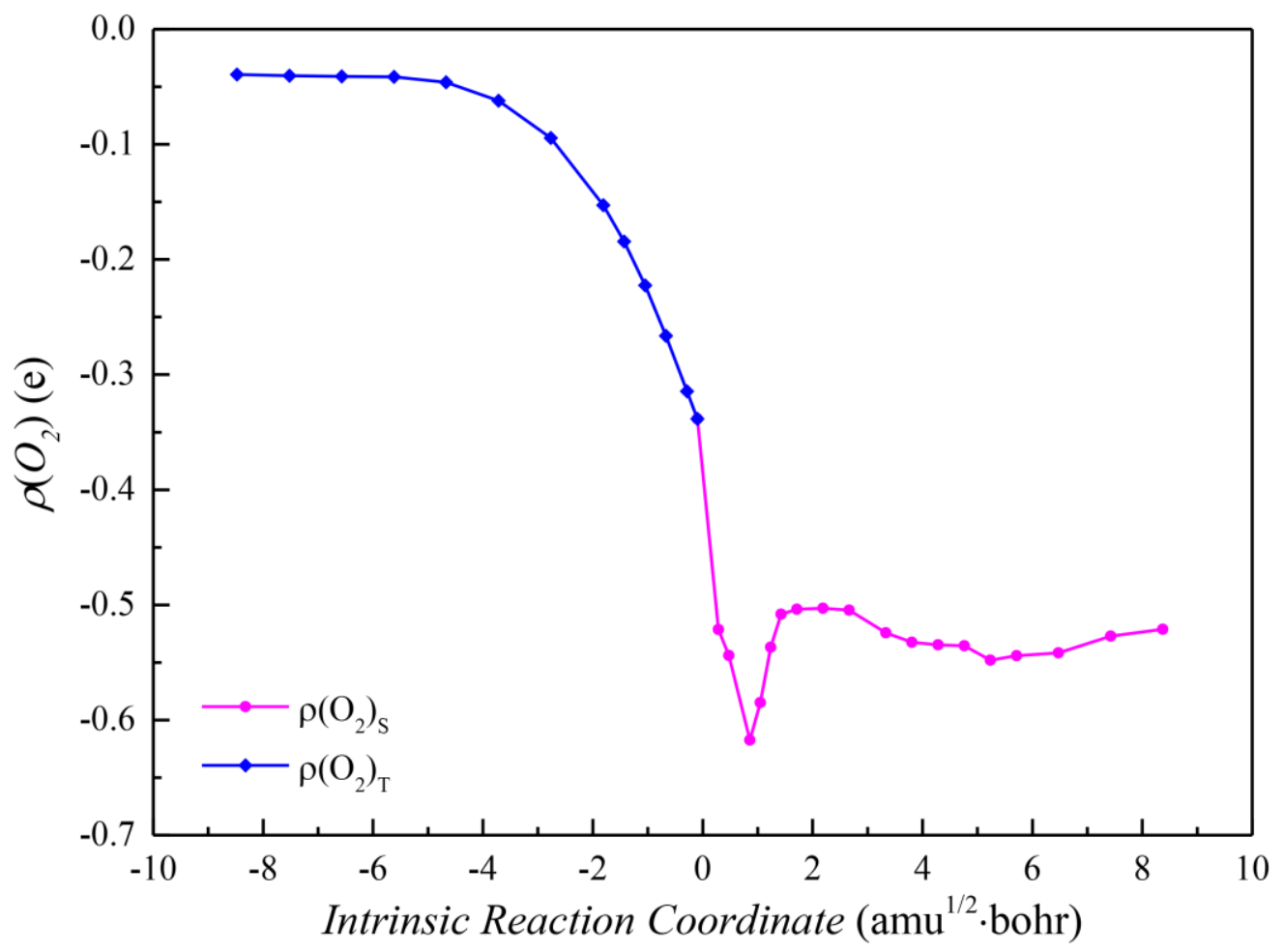

Figure S16. The Mulliken charge of $\mathrm{O}_{2}$ moiety in the reaction path calculated by MS-CASPT2//CAM-B3LYP method. 
Table S3. The MS-CASPT2//SA6-CAS(12,9)/ANO-RCC-VDZP calculated weightings of the electronic configurations (weight $\geq 0.1$ ) at the ISC point in the $\mathrm{S}_{0}$ and $\mathrm{T}_{1}$ states.

\begin{tabular}{clll}
\hline State & Conf. & Coef. & Weight \\
\hline $\mathrm{S}_{0}$ & 22222000 & -0.83705 & 0.700654 \\
\hline \multirow{2}{*}{$\mathrm{T}_{1}$} & $2222 \mathrm{u} 2 \mathrm{u} 00$ & -0.842306 & 0.70948 \\
& $22 \mathrm{u} 22 \mathrm{u} 00$ & -0.334620 & 0.111971 \\
& $222 \mathrm{uu} 2200$ & 0.320286 & 0.102583 \\
\hline
\end{tabular}

\section{Absolute energies and available imaginary frequencies of all optimized structures}

Table S4. Absolute energies (in Hartree) and available imaginary frequencies (in $\mathrm{cm}^{-1}$ ) of optimized structures calculated at (U)CAM-B3LYP/6-311G** level. $E_{e}=$ Electronic energy, $G_{298}=$ Gibbs free energy, $I F=$ Imaginary frequency.

\begin{tabular}{|c|c|c|c|c|}
\hline Environment & Molecules & $E_{e}$ & $G_{298}$ & $I F$ \\
\hline \multirow{5}{*}{ Gas } & ${ }^{1}\left(\mathrm{LF}^{-}\right.$red $\left.\ldots \mathrm{O}_{2}\right)$ & -1022.8919541 & -1022.684282 & -- \\
\hline & ${ }^{3}\left(\mathrm{LF}^{-}\right.$red $\left.\ldots \mathrm{O}_{2}\right)$ & -1022.9056728 & -1022.700711 & -- \\
\hline & ${ }^{1} \mathrm{LFOO}_{\mathrm{H}}^{-}$ & -1022.9115256 & -1022.698281 & -- \\
\hline & ${ }^{3} \mathrm{LFOO}_{\mathrm{H}}^{-}$ & -1022.8400825 & -1022.631493 & -- \\
\hline & $\mathrm{TS}_{\mathrm{S}}$ & -1022.8780912 & -1022.668146 & $602 \mathrm{i}$ \\
\hline \multirow{6}{*}{ Benzene } & ${ }^{1}\left(\mathrm{LF}^{-}\right.$red $\left.\ldots \mathrm{O}_{2}\right)$ & -1022.9327177 & -1022.724967 & -- \\
\hline & ${ }^{3}\left(\mathrm{LF}^{-}\right.$red $\left.\ldots \mathrm{O}_{2}\right)$ & -1022.9484468 & -1022.742569 & -- \\
\hline & ${ }^{1} \mathrm{LFOO}_{\mathrm{H}}^{-}$ & -1022.9548147 & -1022.740805 & -- \\
\hline & ${ }^{3} \mathrm{LFOO}_{\mathrm{H}}^{-}$ & -1022.8859069 & -1022.676930 & -- \\
\hline & ${ }^{1} \mathrm{LF}_{\mathrm{HOO}} \mathrm{OO}^{-}$ & -1022.9437402 & -1022.730315 & -- \\
\hline & $\mathrm{TS}_{\mathrm{S}}$ & -1022.9239519 & -1022.713973 & $645 \mathrm{i}$ \\
\hline
\end{tabular}




\begin{tabular}{|c|c|c|c|c|}
\hline \multirow{6}{*}{ DMSO } & ${ }^{1}\left(\mathrm{LF}^{-}{ }_{\text {red }} \ldots \mathrm{O}_{2}\right)$ & -1022.9710500 & -1022.764493 & -- \\
\hline & ${ }^{3}\left(\mathrm{LF}^{-}{ }_{\text {red }} \ldots \mathrm{O}_{2}\right)$ & -1022.9871253 & -1022.782244 & -- \\
\hline & ${ }^{1} \mathrm{LFOO}_{\mathrm{H}}{ }^{-}$ & -1022.9944812 & -1022.781867 & -- \\
\hline & ${ }^{3} \mathrm{LFOO}_{\mathrm{H}}^{-}$ & -1022.9282404 & -1022.720091 & -- \\
\hline & ${ }^{1} \mathrm{LF}_{\mathrm{H}} \mathrm{OO}^{-}$ & -1022.9881194 & -1022.774914 & -- \\
\hline & $\mathrm{TS}_{\mathrm{S}}$ & -1022.9656915 & -1022.755326 & $394 \mathrm{i}$ \\
\hline \multirow{6}{*}{ Water } & ${ }^{1}\left(\mathrm{LF}^{-}\right.$red $\left.\ldots \mathrm{O}_{2}\right)$ & -1022.9718278 & -1022.765265 & -- \\
\hline & ${ }^{3}\left(\mathrm{LF}^{-}{ }_{\text {red }} \ldots \mathrm{O}_{2}\right)$ & -1022.9879108 & -1022.783020 & -- \\
\hline & ${ }^{1} \mathrm{LFOO}_{\mathrm{H}}^{-}$ & -1022.9952840 & -1022.782691 & -- \\
\hline & ${ }^{3} \mathrm{LFOO}_{\mathrm{H}}^{-}$ & -1022.9291124 & -1022.720973 & -- \\
\hline & ${ }^{1} \mathrm{LF}_{\mathrm{H}} \mathrm{OO}^{-}$ & -1022.9890874 & -1022.775897 & -- \\
\hline & $\mathrm{TS}_{\mathrm{S}}$ & -1022.9665393 & -1022.756172 & $390 \mathrm{i}$ \\
\hline
\end{tabular}

Table S5. Absolute energies (in Hartree) and available imaginary frequencies (in $\mathrm{cm}^{-1}$ ) of optimized structures calculated by four different sets of DFT methods in the gas phase. $E_{e}=$ Electronic energy, $G_{298}=$ Gibbs free energy, $I F=$ Imaginary frequency.

\begin{tabular}{|c|c|c|c|c|}
\hline Methods & Molecules & $\boldsymbol{E}_{e}$ & $G_{298}$ & IF \\
\hline \multirow{3}{*}{ (U)CAM-B3LYP/6-311G** } & ${ }^{1}\left(\mathrm{LF}^{-}{ }_{\text {red }} \ldots \mathrm{O}_{2}\right)$ & -1022.8919541 & -1022.684282 & -- \\
\hline & $\mathrm{TS}_{\mathrm{S}}$ & -1022.8780912 & -1022.668146 & $602 \mathrm{i}$ \\
\hline & ${ }^{1} \mathrm{LFOO}_{\mathrm{H}}^{-}$ & -1022.9115256 & -1022.698281 & -- \\
\hline \multirow{3}{*}{ (U)CAM-B3LYP/6-311+G** } & ${ }^{1}\left(\mathrm{LF}_{\text {red }}^{-} \ldots \mathrm{O}_{2}\right)$ & -1022.9211303 & -1022.714953 & -- \\
\hline & $\mathrm{TS}_{\mathrm{S}}$ & -1022.9101037 & -1022.700450 & $569 \mathrm{i}$ \\
\hline & ${ }^{1} \mathrm{LFOO}_{\mathrm{H}}^{-}$ & -1022.9413786 & -1022.728992 & -- \\
\hline \multirow{3}{*}{ (U)CAM-B3LYP/6-311++G** } & ${ }^{1}\left(\mathrm{LF}_{\text {red }}^{-} \ldots \mathrm{O}_{2}\right)$ & -1022.9212970 & -1022.715097 & -- \\
\hline & $\mathrm{TS}_{\mathrm{S}}$ & -1022.9103706 & -1022.700648 & $569 \mathrm{i}$ \\
\hline & ${ }^{1} \mathrm{LFOO}_{\mathrm{H}}^{-}$ & -1022.9416714 & -1022.729274 & -- \\
\hline \multirow{3}{*}{ (U)wB97XD /6-311G** } & ${ }^{1}\left(\mathrm{LF}_{\text {red }}^{-} \ldots \mathrm{O}_{2}\right)$ & -1023.0196396 & -1022.809976 & -- \\
\hline & $\mathrm{TS}_{\mathrm{S}}$ & -1023.0055953 & -1022.794258 & $553 \mathrm{i}$ \\
\hline & ${ }^{1} \mathrm{LFOO}_{\mathrm{H}}^{-}$ & -1023.0385722 & -1022.825759 & -- \\
\hline
\end{tabular}


Table S6. Absolute energies (in Hartree) of the optimized ISC point in the $\mathrm{S}_{0}$ state and $\mathrm{T}_{1}$ states calculated at CASSCF and MS-CASPT2 level respectively.

\begin{tabular}{ccc}
\hline Methods & ISC $_{\mathrm{S}}$ & ISC $_{\mathrm{T}}$ \\
\hline CASSCF/6-31G* & -1017.08002869 & -1017.08002875 \\
MS-CASPT2//CASSCF/ANO-RCC-VDZP & -1020.86019744 & -1020.86518765 \\
\hline
\end{tabular}

\section{The XYZ Cartesian Coordinates of Key Structures Reported (unit: Ångstrom)}

The XYZ coordinates of stationary points computed at (U)CAM-B3LYP/6-311G** level ${ }^{1}\left(\mathrm{LF}^{-}{ }^{-}{ }^{\cdots} \mathrm{O}_{2}\right)$ in the gas phase

$\begin{array}{lrrr}\mathrm{O} & 2.680567000 & -2.417243000 & -0.605662000 \\ \mathrm{C} & 2.558890000 & -1.211983000 & -0.391048000 \\ \mathrm{~N} & 3.651482000 & -0.389295000 & -0.170532000 \\ \mathrm{H} & 4.560573000 & -0.825137000 & -0.179278000 \\ \mathrm{C} & 3.628635000 & 0.993320000 & 0.059514000 \\ \mathrm{O} & 4.689801000 & 1.578377000 & 0.220215000 \\ \mathrm{~N} & 2.406527000 & 1.595386000 & 0.083212000 \\ \mathrm{C} & 1.325880000 & 0.858501000 & -0.102488000 \\ \mathrm{C} & 1.321640000 & -0.511987000 & -0.334507000 \\ \mathrm{~N} & 0.147347000 & -1.223896000 & -0.593721000 \\ \mathrm{C} & -1.064077000 & -0.609778000 & -0.352071000 \\ \mathrm{C} & -2.254851000 & -1.322492000 & -0.360771000 \\ \mathrm{H} & -2.202181000 & -2.394208000 & -0.521862000 \\ \mathrm{C} & -3.493462000 & -0.714441000 & -0.170146000 \\ \mathrm{C} & -4.750913000 & -1.543022000 & -0.188309000 \\ \mathrm{H} & -5.440232000 & -1.219363000 & -0.975107000 \\ \mathrm{H} & -5.295802000 & -1.472950000 & 0.758626000 \\ \mathrm{H} & -4.520810000 & -2.595330000 & -0.360659000 \\ \mathrm{C} & -3.539939000 & 0.661076000 & 0.036902000 \\ \mathrm{C} & -4.849904000 & 1.374205000 & 0.246751000 \\ \mathrm{H} & -4.691305000 & 2.442912000 & 0.400158000 \\ \mathrm{H} & -5.387605000 & 0.991135000 & 1.120500000 \\ \mathrm{H} & -5.520668000 & 1.259566000 & -0.611472000 \\ \mathrm{C} & -2.342838000 & 1.382424000 & 0.059976000 \\ \mathrm{H} & -2.390352000 & 2.447779000 & 0.239995000 \\ \mathrm{C} & -1.103779000 & 0.782126000 & -0.123661000 \\ \mathrm{~N} & 0.089516000 & 1.516414000 & -0.087116000\end{array}$




$\begin{array}{lrrr}\mathrm{C} & 0.046470000 & 2.939719000 & 0.151627000 \\ \mathrm{H} & 1.073026000 & 3.294274000 & 0.150950000 \\ \mathrm{H} & -0.412199000 & 3.168623000 & 1.121435000 \\ \mathrm{H} & 0.227500000 & -2.204355000 & -0.344698000 \\ \mathrm{O} & 0.415244000 & -2.452337000 & 1.834757000 \\ \mathrm{O} & 1.108476000 & -1.487510000 & 2.165529000 \\ \mathrm{H} & -0.527699000 & 3.446742000 & -0.631191000\end{array}$

\section{${ }^{3}\left(\mathrm{LF}^{-}{ }^{-}{ }^{\cdots}{ }^{\cdots} \mathrm{O}_{2}\right)$ in the gas phase}

$\begin{array}{lrrr}\mathrm{O} & 2.667447000 & -2.435949000 & -0.432524000 \\ \mathrm{C} & 2.551642000 & -1.213451000 & -0.291468000 \\ \mathrm{~N} & 3.641835000 & -0.396131000 & -0.033079000 \\ \mathrm{H} & 4.543777000 & -0.842122000 & 0.029161000 \\ \mathrm{C} & 3.624582000 & 0.996423000 & 0.143811000 \\ \mathrm{O} & 4.686593000 & 1.572629000 & 0.343370000 \\ \mathrm{~N} & 2.411398000 & 1.608158000 & 0.084333000 \\ \mathrm{C} & 1.337339000 & 0.868508000 & -0.154826000 \\ \mathrm{C} & 1.335752000 & -0.496130000 & -0.361841000 \\ \mathrm{~N} & 0.153650000 & -1.200579000 & -0.680056000 \\ \mathrm{C} & -1.056032000 & -0.604254000 & -0.405950000 \\ \mathrm{C} & -2.245146000 & -1.318663000 & -0.373380000 \\ \mathrm{H} & -2.197403000 & -2.392246000 & -0.526688000 \\ \mathrm{C} & -3.482650000 & -0.709028000 & -0.160337000 \\ \mathrm{C} & -4.738287000 & -1.540969000 & -0.146114000 \\ \mathrm{H} & -5.443207000 & -1.227223000 & -0.923229000 \\ \mathrm{H} & -5.266522000 & -1.462741000 & 0.809772000 \\ \mathrm{H} & -4.509204000 & -2.594678000 & -0.312458000 \\ \mathrm{C} & -3.527537000 & 0.665123000 & 0.037671000 \\ \mathrm{C} & -4.832943000 & 1.379441000 & 0.272318000 \\ \mathrm{H} & -4.670870000 & 2.449627000 & 0.412123000 \\ \mathrm{H} & -5.350597000 & 1.005456000 & 1.162302000 \\ \mathrm{H} & -5.524728000 & 1.258107000 & -0.568453000 \\ \mathrm{C} & -2.328384000 & 1.389460000 & 0.025464000 \\ \mathrm{H} & -2.374278000 & 2.456788000 & 0.195012000 \\ \mathrm{C} & -1.094591000 & 0.794021000 & -0.191711000 \\ \mathrm{~N} & 0.098014000 & 1.533902000 & -0.215047000 \\ \mathrm{C} & 0.053396000 & 2.960413000 & -0.005623000 \\ \mathrm{H} & 1.077083000 & 3.321365000 & -0.044212000 \\ \mathrm{H} & -0.373432000 & 3.215081000 & 0.973825000 \\ \mathrm{H} & 0.242244000 & -2.197392000 & -0.536183000 \\ \mathrm{O} & 0.352812000 & -2.558575000 & 1.884645000 \\ \mathrm{O} & 0.932026000 & -1.591665000 & 2.315544000 \\ \mathrm{H} & -0.550823000 & 3.444547000 & -0.780327000\end{array}$

${ }^{1} \mathrm{LFOO}_{\mathrm{H}^{-}}$in the gas phase 


$\begin{array}{lrrr}\mathrm{O} & 2.679911000 & -2.447224000 & -0.717385000 \\ \mathrm{C} & 2.540601000 & -1.280604000 & -0.448854000 \\ \mathrm{~N} & 3.596100000 & -0.398721000 & -0.509404000 \\ \mathrm{H} & 4.504852000 & -0.792660000 & -0.704300000 \\ \mathrm{C} & 3.567219000 & 0.986498000 & -0.296610000 \\ \mathrm{O} & 4.615793000 & 1.597140000 & -0.307353000 \\ \mathrm{~N} & 2.344368000 & 1.582849000 & -0.147256000 \\ \mathrm{C} & 1.270365000 & 0.856284000 & -0.019694000 \\ \mathrm{C} & 1.241277000 & -0.656272000 & 0.061454000 \\ \mathrm{~N} & 0.136224000 & -1.295669000 & -0.482687000 \\ \mathrm{C} & -1.037073000 & -0.642682000 & -0.321910000 \\ \mathrm{C} & -2.259617000 & -1.312366000 & -0.530653000 \\ \mathrm{H} & -2.190232000 & -2.352839000 & -0.829353000 \\ \mathrm{C} & -3.492852000 & -0.714285000 & -0.358385000 \\ \mathrm{C} & -4.753144000 & -1.509696000 & -0.582698000 \\ \mathrm{H} & -5.386042000 & -1.057887000 & -1.353662000 \\ \mathrm{H} & -5.359821000 & -1.573271000 & 0.326759000 \\ \mathrm{H} & -4.517104000 & -2.527011000 & -0.897478000 \\ \mathrm{C} & -3.561721000 & 0.632435000 & 0.035454000 \\ \mathrm{C} & -4.884096000 & 1.318558000 & 0.256058000 \\ \mathrm{H} & -4.735815000 & 2.361942000 & 0.541936000 \\ \mathrm{H} & -5.469941000 & 0.842038000 & 1.050179000 \\ \mathrm{H} & -5.510650000 & 1.310723000 & -0.643035000 \\ \mathrm{C} & -2.371843000 & 1.327435000 & 0.209628000 \\ \mathrm{H} & -2.423446000 & 2.370945000 & 0.495139000 \\ \mathrm{C} & -1.133191000 & 0.723523000 & 0.027508000 \\ \mathrm{~N} & 0.068988000 & 1.461895000 & 0.086071000 \\ \mathrm{C} & 0.009078000 & 2.912507000 & 0.153066000 \\ \mathrm{H} & 1.024637000 & 3.288200000 & 0.087823000 \\ \mathrm{H} & -0.443190000 & 3.226672000 & 1.096721000 \\ \mathrm{H} & 0.433875000 & -2.402451000 & 1.021360000 \\ \mathrm{O} & 0.991004000 & -2.216181000 & 1.814515000 \\ \mathrm{H} & 1.317440000 & -0.845809000 & 1.554323000 \\ & -0.589411000 & 3.302486000 & -0.673270000\end{array}$

\section{${ }^{3} \mathrm{LFOOH}^{-}$in the gas phase}

$\begin{array}{llrl}\mathrm{O} & 2.462241000 & -2.367858000 & -1.051798000 \\ \mathrm{C} & 2.442432000 & -1.238813000 & -0.604651000 \\ \mathrm{~N} & 3.493548000 & -0.378098000 & -0.703522000 \\ \mathrm{H} & 4.345540000 & -0.749923000 & -1.095799000 \\ \mathrm{C} & 3.521708000 & 1.016858000 & -0.399481000 \\ \mathrm{O} & 4.605695000 & 1.588451000 & -0.494942000 \\ \mathrm{~N} & 2.347308000 & 1.578830000 & -0.088034000 \\ \mathrm{C} & 1.265827000 & 0.808858000 & 0.026009000 \\ \mathrm{C} & 1.264717000 & -0.649314000 & 0.174961000 \\ & & & \mathrm{~S} 22\end{array}$




$\begin{array}{lrrr}\mathrm{N} & 0.044043000 & -1.357589000 & -0.128096000 \\ \mathrm{C} & -1.072212000 & -0.649952000 & -0.145979000 \\ \mathrm{C} & -2.302755000 & -1.339081000 & -0.335768000 \\ \mathrm{H} & -2.217138000 & -2.408576000 & -0.490447000 \\ \mathrm{C} & -3.528751000 & -0.722499000 & -0.323639000 \\ \mathrm{C} & -4.790613000 & -1.515844000 & -0.537305000 \\ \mathrm{H} & -5.348791000 & -1.168238000 & -1.412821000 \\ \mathrm{H} & -5.468597000 & -1.445917000 & 0.319569000 \\ \mathrm{H} & -4.557508000 & -2.570221000 & -0.690383000 \\ \mathrm{C} & -3.577742000 & 0.673620000 & -0.095786000 \\ \mathrm{C} & -4.899336000 & 1.390185000 & -0.057466000 \\ \mathrm{H} & -4.761875000 & 2.456300000 & 0.126587000 \\ \mathrm{H} & -5.548797000 & 0.994416000 & 0.729967000 \\ \mathrm{H} & -5.444750000 & 1.279503000 & -1.000199000 \\ \mathrm{C} & -2.403350000 & 1.389756000 & 0.090010000 \\ \mathrm{H} & -2.470088000 & 2.456032000 & 0.258012000 \\ \mathrm{C} & -1.139514000 & 0.782392000 & 0.057309000 \\ \mathrm{~N} & 0.010546000 & 1.473119000 & 0.216990000 \\ \mathrm{C} & -0.000624000 & 2.899480000 & 0.448618000 \\ \mathrm{H} & 1.036401000 & 3.216813000 & 0.513220000 \\ \mathrm{H} & -0.531573000 & 3.136993000 & 1.376838000 \\ \mathrm{H} & 0.866862000 & -2.664044000 & 1.242315000 \\ \mathrm{O} & 1.573135000 & -2.352899000 & 1.835013000 \\ \mathrm{O} & 1.492316000 & -0.918323000 & 1.612331000 \\ \mathrm{H} & -0.485767000 & 3.432078000 & -0.377626000\end{array}$

\section{The TSs in the gas phase}

$\begin{array}{lrrr}\mathrm{O} & 2.660933000 & -2.386302000 & -0.939308000 \\ \mathrm{C} & 2.535208000 & -1.236409000 & -0.569102000 \\ \mathrm{~N} & 3.617374000 & -0.379692000 & -0.473765000 \\ \mathrm{H} & 4.529203000 & -0.778698000 & -0.636136000 \\ \mathrm{C} & 3.588853000 & 0.970788000 & -0.117601000 \\ \mathrm{O} & 4.637399000 & 1.579073000 & -0.020464000 \\ \mathrm{~N} & 2.358145000 & 1.550023000 & 0.057962000 \\ \mathrm{C} & 1.282010000 & 0.810552000 & 0.008090000 \\ \mathrm{C} & 1.283775000 & -0.612307000 & -0.148068000 \\ \mathrm{~N} & 0.128128000 & -1.355029000 & -0.295325000 \\ \mathrm{C} & -1.083712000 & -0.665926000 & -0.204338000 \\ \mathrm{C} & -2.277910000 & -1.364615000 & -0.319866000 \\ \mathrm{H} & -2.212069000 & -2.436272000 & -0.472899000 \\ \mathrm{C} & -3.519195000 & -0.747320000 & -0.240872000 \\ \mathrm{C} & -4.780312000 & -1.562004000 & -0.356241000 \\ \mathrm{H} & -5.398555000 & -1.238454000 & -1.199908000 \\ \mathrm{H} & -5.400096000 & -1.477575000 & 0.542134000 \\ \mathrm{H} & -4.547237000 & -2.617672000 & -0.499999000 \\ & & & \mathrm{~S} 23\end{array}$




$\begin{array}{lrrr}\mathrm{C} & -3.567976000 & 0.637041000 & -0.060151000 \\ \mathrm{C} & -4.885236000 & 1.362648000 & 0.022481000 \\ \mathrm{H} & -4.732999000 & 2.432082000 & 0.176328000 \\ \mathrm{H} & -5.500862000 & 0.992676000 & 0.848596000 \\ \mathrm{H} & -5.475549000 & 1.239510000 & -0.891362000 \\ \mathrm{C} & -2.375983000 & 1.346720000 & 0.047587000 \\ \mathrm{H} & -2.426539000 & 2.416400000 & 0.198974000 \\ \mathrm{C} & -1.133070000 & 0.720649000 & -0.010954000 \\ \mathrm{~N} & 0.061727000 & 1.440695000 & 0.121273000 \\ \mathrm{C} & 0.017979000 & 2.867404000 & 0.366990000 \\ \mathrm{H} & 1.044001000 & 3.209168000 & 0.459527000 \\ \mathrm{H} & -0.530802000 & 3.079284000 & 1.289139000 \\ \mathrm{H} & 0.193555000 & -2.093191000 & 0.482732000 \\ \mathrm{O} & 0.737889000 & -2.020431000 & 1.969293000 \\ \mathrm{O} & 1.633018000 & -1.091637000 & 1.761163000 \\ \mathrm{H} & -0.467922000 & 3.392399000 & -0.461636000\end{array}$

\section{${ }^{1}\left(\mathrm{LF}^{-}{ }^{-}{ }^{\prime}{ }^{\cdots} \mathrm{O}_{2}\right)$ in benzene}

$\begin{array}{lrrr}\mathrm{O} & 2.672117000 & -2.416965000 & -0.517309000 \\ \mathrm{C} & 2.549403000 & -1.199946000 & -0.330786000 \\ \mathrm{~N} & 3.638778000 & -0.393282000 & -0.037968000 \\ \mathrm{H} & 4.541128000 & -0.841547000 & 0.013288000 \\ \mathrm{C} & 3.613835000 & 0.989041000 & 0.177299000 \\ \mathrm{O} & 4.674679000 & 1.567083000 & 0.401474000 \\ \mathrm{~N} & 2.405591000 & 1.603503000 & 0.127092000 \\ \mathrm{C} & 1.327016000 & 0.875329000 & -0.141310000 \\ \mathrm{C} & 1.330528000 & -0.487531000 & -0.383133000 \\ \mathrm{~N} & 0.156461000 & -1.183054000 & -0.738441000 \\ \mathrm{C} & -1.057172000 & -0.591125000 & -0.433091000 \\ \mathrm{C} & -2.241096000 & -1.312835000 & -0.405922000 \\ \mathrm{H} & -2.191809000 & -2.381092000 & -0.590847000 \\ \mathrm{C} & -3.476712000 & -0.714465000 & -0.160571000 \\ \mathrm{C} & -4.730222000 & -1.549027000 & -0.154795000 \\ \mathrm{H} & -5.440399000 & -1.216813000 & -0.918436000 \\ \mathrm{H} & -5.249432000 & -1.491451000 & 0.806637000 \\ \mathrm{H} & -4.502082000 & -2.598090000 & -0.347046000 \\ \mathrm{C} & -3.522385000 & 0.656164000 & 0.077022000 \\ \mathrm{C} & -4.827284000 & 1.357283000 & 0.350125000 \\ \mathrm{H} & -4.670955000 & 2.424859000 & 0.511156000 \\ \mathrm{H} & -5.324856000 & 0.957621000 & 1.239490000 \\ \mathrm{H} & -5.530651000 & 1.247331000 & -0.481448000 \\ \mathrm{C} & -2.328180000 & 1.384199000 & 0.069160000 \\ \mathrm{H} & -2.375025000 & 2.445655000 & 0.271436000 \\ \mathrm{C} & -1.096377000 & 0.795728000 & -0.181972000 \\ \mathrm{~N} & 0.097617000 & 1.540618000 & -0.201734000 \\ & & & \mathrm{~S} 24 \\ & & & \end{array}$




$\begin{array}{lrrr}\mathrm{C} & 0.045005000 & 2.969958000 & 0.006264000 \\ \mathrm{H} & 1.057049000 & 3.351353000 & -0.079725000 \\ \mathrm{H} & -0.340863000 & 3.223782000 & 1.001414000 \\ \mathrm{H} & 0.235747000 & -2.180196000 & -0.586062000 \\ \mathrm{O} & 0.362062000 & -2.550583000 & 1.741681000 \\ \mathrm{O} & 0.939203000 & -1.582548000 & 2.187281000 \\ \mathrm{H} & -0.597759000 & 3.438295000 & -0.744376000\end{array}$

\section{${ }^{3}\left(\mathrm{LF}^{-}{ }^{-}{ }^{\cdots} \mathrm{O}_{2}\right)$ in benzene}

$\begin{array}{lrrr}\mathrm{O} & 2.666064000 & -2.430065000 & -0.395134000 \\ \mathrm{C} & 2.545723000 & -1.204021000 & -0.262284000 \\ \mathrm{~N} & 3.632224000 & -0.394356000 & 0.035618000 \\ \mathrm{H} & 4.528936000 & -0.846812000 & 0.130507000 \\ \mathrm{C} & 3.611591000 & 0.995375000 & 0.202370000 \\ \mathrm{O} & 4.672121000 & 1.573742000 & 0.432420000 \\ \mathrm{~N} & 2.409349000 & 1.613037000 & 0.104622000 \\ \mathrm{C} & 1.334649000 & 0.879531000 & -0.174356000 \\ \mathrm{C} & 1.338279000 & -0.484951000 & -0.385107000 \\ \mathrm{~N} & 0.163191000 & -1.182760000 & -0.756890000 \\ \mathrm{C} & -1.049624000 & -0.590181000 & -0.447362000 \\ \mathrm{C} & -2.232606000 & -1.311680000 & -0.395214000 \\ \mathrm{H} & -2.184886000 & -2.383367000 & -0.560831000 \\ \mathrm{C} & -3.467790000 & -0.708907000 & -0.153188000 \\ \mathrm{C} & -4.720610000 & -1.544273000 & -0.124767000 \\ \mathrm{H} & -5.435093000 & -1.227393000 & -0.890971000 \\ \mathrm{H} & -5.235316000 & -1.469216000 & 0.837954000 \\ \mathrm{H} & -4.492512000 & -2.596636000 & -0.298515000 \\ \mathrm{C} & -3.512783000 & 0.665264000 & 0.057879000 \\ \mathrm{C} & -4.816285000 & 1.371469000 & 0.325321000 \\ \mathrm{H} & -4.658775000 & 2.441586000 & 0.467516000 \\ \mathrm{H} & -5.310088000 & 0.987404000 & 1.223661000 \\ \mathrm{H} & -5.523832000 & 1.248083000 & -0.500906000 \\ \mathrm{C} & -2.318318000 & 1.393949000 & 0.026692000 \\ \mathrm{H} & -2.364911000 & 2.459472000 & 0.206747000 \\ \mathrm{C} & -1.088481000 & 0.802326000 & -0.224414000 \\ \mathrm{~N} & 0.105483000 & 1.547137000 & -0.273715000 \\ \mathrm{C} & 0.052805000 & 2.978487000 & -0.082391000 \\ \mathrm{H} & 1.064314000 & 3.359636000 & -0.174236000 \\ \mathrm{H} & -0.330569000 & 3.245635000 & 0.910738000 \\ \mathrm{H} & 0.242605000 & -2.177615000 & -0.592339000 \\ \mathrm{H} & 0.297170000 & -2.636883000 & 1.804952000 \\ & 0.812999000 & -1.673539000 & 2.308302000 \\ \mathrm{O} & -0.592071000 & 3.437773000 & -0.836831000\end{array}$

${ }^{1} \mathrm{LFOO}_{\mathrm{H}}{ }^{-}$in benzene 


\begin{tabular}{|c|c|c|c|}
\hline $\mathrm{O}$ & 2.673468000 & -2.436993000 & -0.759488000 \\
\hline $\mathrm{C}$ & 2.542776000 & -1.272243000 & -0.469975000 \\
\hline $\mathrm{N}$ & 3.601517000 & -0.398934000 & -0.507707000 \\
\hline $\mathrm{H}$ & 4.510723000 & -0.790218000 & -0.710300000 \\
\hline $\mathrm{C}$ & 3.568612000 & 0.983074000 & -0.276559000 \\
\hline $\mathrm{O}$ & 4.619527000 & 1.594042000 & -0.275620000 \\
\hline $\mathrm{N}$ & 2.351487000 & 1.580756000 & -0.120628000 \\
\hline $\mathrm{C}$ & 1.268990000 & 0.857214000 & -0.017510000 \\
\hline $\mathrm{C}$ & 1.243598000 & -0.654237000 & 0.042820000 \\
\hline $\mathrm{N}$ & 0.140470000 & -1.295741000 & -0.490351000 \\
\hline $\mathrm{C}$ & -1.038942000 & -0.641567000 & -0.321788000 \\
\hline $\mathrm{C}$ & -2.258636000 & -1.317221000 & -0.516394000 \\
\hline $\mathrm{H}$ & -2.192481000 & -2.361429000 & -0.802576000 \\
\hline $\mathrm{C}$ & -3.492737000 & -0.718648000 & -0.347844000 \\
\hline $\mathrm{C}$ & -4.753529000 & -1.515485000 & -0.559875000 \\
\hline $\mathrm{H}$ & -5.383440000 & -1.073748000 & -1.338153000 \\
\hline $\mathrm{H}$ & -5.360136000 & -1.560178000 & 0.349938000 \\
\hline $\mathrm{H}$ & -4.521406000 & -2.538540000 & -0.857253000 \\
\hline $\mathrm{C}$ & -3.559190000 & 0.633756000 & 0.029981000 \\
\hline $\mathrm{C}$ & -4.882015000 & 1.322699000 & 0.239230000 \\
\hline $\mathrm{H}$ & -4.736532000 & 2.369843000 & 0.510104000 \\
\hline $\mathrm{H}$ & -5.466692000 & 0.854240000 & 1.037782000 \\
\hline $\mathrm{H}$ & -5.504043000 & 1.298449000 & -0.661579000 \\
\hline $\mathrm{C}$ & -2.370881000 & 1.330292000 & 0.198283000 \\
\hline $\mathrm{H}$ & -2.423044000 & 2.375809000 & 0.473906000 \\
\hline $\mathrm{C}$ & -1.131154000 & 0.722894000 & 0.021320000 \\
\hline $\mathrm{N}$ & 0.071502000 & 1.462235000 & 0.082255000 \\
\hline $\mathrm{C}$ & 0.005803000 & 2.915236000 & 0.160931000 \\
\hline $\mathrm{H}$ & 1.018286000 & 3.299188000 & 0.117114000 \\
\hline $\mathrm{H}$ & -0.462246000 & 3.216860000 & 1.099622000 \\
\hline $\mathrm{H}$ & 0.410432000 & -2.384721000 & 0.994543000 \\
\hline $\mathrm{O}$ & 0.972350000 & -2.225663000 & 1.793484000 \\
\hline $\mathrm{O}$ & 1.339209000 & -0.863387000 & 1.548504000 \\
\hline $\mathrm{H}$ & -0.579103000 & 3.308867000 & -0.672212000 \\
\hline \multicolumn{4}{|c|}{${ }^{3} \mathrm{LFOOH}^{-}$in benzene } \\
\hline $\mathrm{O}$ & 2.425230000 & -2.319141000 & -1.170423000 \\
\hline $\mathrm{C}$ & 2.425338000 & -1.217469000 & -0.654140000 \\
\hline $\mathrm{N}$ & 3.492600000 & -0.373751000 & -0.704263000 \\
\hline $\mathrm{H}$ & 4.339424000 & -0.736654000 & -1.116733000 \\
\hline $\mathrm{C}$ & 3.530188000 & 1.000745000 & -0.333280000 \\
\hline $\mathrm{O}$ & 4.626207000 & 1.565369000 & -0.376473000 \\
\hline $\mathrm{N}$ & 2.360314000 & 1.567312000 & -0.023843000 \\
\hline $\mathrm{C}$ & 1.264232000 & 0.810333000 & 0.044815000 \\
\hline $\mathrm{C}$ & 1.262781000 & -0.651605000 & $\begin{array}{l}0.161746000 \\
\text { S26 }\end{array}$ \\
\hline
\end{tabular}




$\begin{array}{lrrr}\mathrm{N} & 0.042747000 & -1.359775000 & -0.118658000 \\ \mathrm{C} & -1.075098000 & -0.651833000 & -0.135357000 \\ \mathrm{C} & -2.306231000 & -1.344108000 & -0.315342000 \\ \mathrm{H} & -2.224087000 & -2.415160000 & -0.459436000 \\ \mathrm{C} & -3.530017000 & -0.727444000 & -0.310034000 \\ \mathrm{C} & -4.793273000 & -1.520373000 & -0.512202000 \\ \mathrm{H} & -5.349985000 & -1.178945000 & -1.390041000 \\ \mathrm{H} & -5.467479000 & -1.435573000 & 0.345306000 \\ \mathrm{H} & -4.564724000 & -2.577016000 & -0.653202000 \\ \mathrm{C} & -3.576828000 & 0.674218000 & -0.101356000 \\ \mathrm{C} & -4.896470000 & 1.392370000 & -0.073317000 \\ \mathrm{H} & -4.760466000 & 2.459821000 & 0.100700000 \\ \mathrm{H} & -5.546199000 & 1.002028000 & 0.715680000 \\ \mathrm{H} & -5.437965000 & 1.270160000 & -1.016141000 \\ \mathrm{C} & -2.400842000 & 1.389908000 & 0.075977000 \\ \mathrm{H} & -2.467401000 & 2.457907000 & 0.230337000 \\ \mathrm{C} & -1.140334000 & 0.779822000 & 0.056165000 \\ \mathrm{~N} & 0.012572000 & 1.475384000 & 0.221499000 \\ \mathrm{C} & -0.007856000 & 2.907910000 & 0.427161000 \\ \mathrm{H} & 1.023406000 & 3.236424000 & 0.506899000 \\ \mathrm{H} & -0.553615000 & 3.158807000 & 1.341713000 \\ \mathrm{H} & 0.846748000 & -2.667108000 & 1.263361000 \\ \mathrm{O} & 1.612768000 & -2.377809000 & 1.789940000 \\ \mathrm{O} & 1.538122000 & -0.940935000 & 1.592857000 \\ \mathrm{H} & -0.481270000 & 3.422677000 & -0.415907000\end{array}$

\section{${ }^{1} \mathrm{LF}_{\mathrm{HOO}} \mathrm{OO}^{-}$in benzene}

$\begin{array}{lrrr}\mathrm{O} & 2.660534000 & -2.255484000 & -1.183376000 \\ \mathrm{C} & 2.563863000 & -1.199255000 & -0.611719000 \\ \mathrm{~N} & 3.652597000 & -0.395795000 & -0.365147000 \\ \mathrm{H} & 4.568550000 & -0.775249000 & -0.559082000 \\ \mathrm{C} & 3.609467000 & 0.938703000 & 0.043741000 \\ \mathrm{O} & 4.648372000 & 1.519722000 & 0.275840000 \\ \mathrm{~N} & 2.382986000 & 1.561926000 & 0.093352000 \\ \mathrm{C} & 1.292114000 & 0.868589000 & -0.012528000 \\ \mathrm{C} & 1.273916000 & -0.643039000 & 0.000414000 \\ \mathrm{~N} & 0.150846000 & -1.205959000 & -0.691869000 \\ \mathrm{C} & -1.065871000 & -0.594886000 & -0.412968000 \\ \mathrm{C} & -2.262310000 & -1.300649000 & -0.485415000 \\ \mathrm{H} & -2.212113000 & -2.359042000 & -0.716335000 \\ \mathrm{C} & -3.494281000 & -0.699025000 & -0.270644000 \\ \mathrm{C} & -4.757335000 & -1.513593000 & -0.355928000 \\ \mathrm{H} & -5.439974000 & -1.119432000 & -1.114562000 \\ \mathrm{H} & -5.301210000 & -1.509584000 & 0.593231000 \\ \mathrm{H} & -4.536096000 & -2.550462000 & -0.609636000 \\ & & & \mathrm{~S} 27\end{array}$




$\begin{array}{lrrr}\mathrm{C} & -3.540065000 & 0.667210000 & 0.032029000 \\ \mathrm{C} & -4.851316000 & 1.365365000 & 0.279140000 \\ \mathrm{H} & -4.695355000 & 2.421500000 & 0.502742000 \\ \mathrm{H} & -5.391986000 & 0.923483000 & 1.121467000 \\ \mathrm{H} & -5.513936000 & 1.303285000 & -0.589477000 \\ \mathrm{C} & -2.347036000 & 1.376646000 & 0.109752000 \\ \mathrm{H} & -2.388320000 & 2.428679000 & 0.357251000 \\ \mathrm{C} & -1.116461000 & 0.766252000 & -0.104890000 \\ \mathrm{~N} & 0.096036000 & 1.493923000 & -0.041758000 \\ \mathrm{C} & 0.045619000 & 2.946282000 & 0.055009000 \\ \mathrm{H} & 1.057737000 & 3.322697000 & -0.038740000 \\ \mathrm{H} & -0.361851000 & 3.251942000 & 1.021351000 \\ \mathrm{H} & 0.176735000 & -2.171228000 & -0.317229000 \\ \mathrm{O} & 0.994646000 & -2.433249000 & 1.403351000 \\ \mathrm{O} & 1.269818000 & -0.997170000 & 1.366192000 \\ \mathrm{H} & -0.581625000 & 3.346942000 & -0.741965000\end{array}$

\section{The TSs in benzene}

$\begin{array}{lrrr}\mathrm{O} & 2.655759000 & -2.385581000 & -0.910346000 \\ \mathrm{C} & 2.536714000 & -1.228387000 & -0.551137000 \\ \mathrm{~N} & 3.622111000 & -0.382921000 & -0.443225000 \\ \mathrm{H} & 4.534876000 & -0.784423000 & -0.599394000 \\ \mathrm{C} & 3.589288000 & 0.967962000 & -0.090844000 \\ \mathrm{O} & 4.642033000 & 1.573595000 & 0.014314000 \\ \mathrm{~N} & 2.365563000 & 1.552985000 & 0.079075000 \\ \mathrm{C} & 1.278782000 & 0.822440000 & 0.007135000 \\ \mathrm{C} & 1.281942000 & -0.597208000 & -0.166519000 \\ \mathrm{~N} & 0.132491000 & -1.338974000 & -0.328790000 \\ \mathrm{C} & -1.085085000 & -0.656522000 & -0.216736000 \\ \mathrm{C} & -2.276721000 & -1.361513000 & -0.323419000 \\ \mathrm{H} & -2.212478000 & -2.433062000 & -0.477923000 \\ \mathrm{C} & -3.517597000 & -0.746706000 & -0.234446000 \\ \mathrm{C} & -4.778181000 & -1.562596000 & -0.338580000 \\ \mathrm{H} & -5.399445000 & -1.242181000 & -1.180296000 \\ \mathrm{H} & -5.391539000 & -1.470293000 & 0.562591000 \\ \mathrm{H} & -4.547033000 & -2.619026000 & -0.476951000 \\ \mathrm{C} & -3.566763000 & 0.639868000 & -0.054186000 \\ \mathrm{C} & -4.885000000 & 1.362189000 & 0.033134000 \\ \mathrm{H} & -4.736441000 & 2.432520000 & 0.180530000 \\ \mathrm{H} & -5.492917000 & 0.992153000 & 0.863968000 \\ \mathrm{H} & -5.478134000 & 1.230039000 & -0.876767000 \\ \mathrm{C} & -2.376690000 & 1.351744000 & 0.047486000 \\ \mathrm{H} & -2.429525000 & 2.421104000 & 0.197757000 \\ \mathrm{C} & -1.132932000 & 0.726943000 & -0.019382000 \\ \mathrm{~N} & 0.064275000 & 1.451744000 & 0.108005000 \\ & & & \mathrm{~S} 28 \\ & & & \end{array}$




$\begin{array}{rrrr}\mathrm{C} & 0.011073000 & 2.884065000 & 0.338883000 \\ \mathrm{H} & 1.031445000 & 3.239889000 & 0.426368000 \\ \mathrm{H} & -0.535119000 & 3.100887000 & 1.260053000 \\ \mathrm{H} & 0.201670000 & -2.113978000 & 0.400833000 \\ \mathrm{O} & 0.743354000 & -2.093767000 & 1.923270000 \\ \mathrm{O} & 1.625311000 & -1.141186000 & 1.774349000 \\ \mathrm{H} & -0.481913000 & 3.391777000 & -0.494539000\end{array}$

\section{${ }^{1}\left(\mathrm{LF}^{-}{ }^{\mathrm{red}} \boldsymbol{\cdots O}_{2}\right)$ in DMSO}

$\begin{array}{lrrr}\mathrm{O} & 2.590701000 & -2.346729000 & -0.664724000 \\ \mathrm{C} & 2.453017000 & -1.129880000 & -0.467790000 \\ \mathrm{~N} & 3.536283000 & -0.323103000 & -0.152580000 \\ \mathrm{H} & 4.441895000 & -0.766536000 & -0.100090000 \\ \mathrm{C} & 3.493543000 & 1.051327000 & 0.076003000 \\ \mathrm{O} & 4.549856000 & 1.640747000 & 0.321376000 \\ \mathrm{~N} & 2.287170000 & 1.659449000 & 0.022221000 \\ \mathrm{C} & 1.208134000 & 0.929267000 & -0.268394000 \\ \mathrm{C} & 1.230260000 & -0.430606000 & -0.531168000 \\ \mathrm{~N} & 0.065345000 & -1.133312000 & -0.911373000 \\ \mathrm{C} & -1.155866000 & -0.558559000 & -0.568375000 \\ \mathrm{C} & -2.328104000 & -1.298873000 & -0.532094000 \\ \mathrm{H} & -2.269861000 & -2.362324000 & -0.740432000 \\ \mathrm{C} & -3.565822000 & -0.721849000 & -0.249794000 \\ \mathrm{C} & -4.808668000 & -1.571157000 & -0.233151000 \\ \mathrm{H} & -5.543383000 & -1.221990000 & -0.964310000 \\ \mathrm{H} & -5.299498000 & -1.544725000 & 0.743920000 \\ \mathrm{H} & -4.576096000 & -2.611263000 & -0.462949000 \\ \mathrm{C} & -3.623532000 & 0.645894000 & 0.015000000 \\ \mathrm{C} & -4.931493000 & 1.324591000 & 0.325507000 \\ \mathrm{H} & -4.785949000 & 2.389279000 & 0.510703000 \\ \mathrm{H} & -5.409935000 & 0.894006000 & 1.210040000 \\ \mathrm{H} & -5.644112000 & 1.223698000 & -0.498397000 \\ \mathrm{C} & -2.440495000 & 1.389623000 & -0.004574000 \\ \mathrm{H} & -2.498180000 & 2.446507000 & 0.216958000 \\ \mathrm{C} & -1.207597000 & 0.819382000 & -0.293741000 \\ \mathrm{~N} & -0.019085000 & 1.582919000 & -0.325228000 \\ \mathrm{C} & -0.093984000 & 3.013158000 & -0.106955000 \\ \mathrm{H} & 0.900572000 & 3.424715000 & -0.234780000 \\ \mathrm{H} & -0.444545000 & 3.255411000 & 0.903007000 \\ \mathrm{H} & 0.148445000 & -2.129897000 & -0.754093000 \\ \mathrm{O} & 0.298736000 & -2.515272000 & 1.614111000 \\ \mathrm{O} & 0.828509000 & -1.527025000 & 2.066798000 \\ \mathrm{H} & -0.776671000 & 3.467181000 & -0.828130000\end{array}$

${ }^{3}\left(\mathrm{LF}^{-}{ }_{\text {red }} \cdots \mathrm{O}_{2}\right)$ in DMSO 


$\begin{array}{lrrr}\mathrm{O} & 2.664695000 & -2.418237000 & -0.376905000 \\ \mathrm{C} & 2.537158000 & -1.190471000 & -0.241107000 \\ \mathrm{~N} & 3.616847000 & -0.389892000 & 0.105606000 \\ \mathrm{H} & 4.508003000 & -0.847749000 & 0.230801000 \\ \mathrm{C} & 3.591013000 & 0.994095000 & 0.274631000 \\ \mathrm{O} & 4.646228000 & 1.572142000 & 0.555399000 \\ \mathrm{~N} & 2.403131000 & 1.620653000 & 0.129629000 \\ \mathrm{C} & 1.330083000 & 0.893960000 & -0.199081000 \\ \mathrm{C} & 1.339953000 & -0.470185000 & -0.413575000 \\ \mathrm{~N} & 0.174470000 & -1.158196000 & -0.847065000 \\ \mathrm{C} & -1.043016000 & -0.572683000 & -0.499334000 \\ \mathrm{C} & -2.218656000 & -1.303407000 & -0.424385000 \\ \mathrm{H} & -2.171143000 & -2.373386000 & -0.600197000 \\ \mathrm{C} & -3.450107000 & -0.708018000 & -0.147471000 \\ \mathrm{C} & -4.698795000 & -1.547492000 & -0.093720000 \\ \mathrm{H} & -5.436804000 & -1.217425000 & -0.830514000 \\ \mathrm{H} & -5.182315000 & -1.486173000 & 0.885456000 \\ \mathrm{H} & -4.475096000 & -2.596186000 & -0.291297000 \\ \mathrm{C} & -3.495288000 & 0.666737000 & 0.076444000 \\ \mathrm{C} & -4.795300000 & 1.364210000 & 0.379716000 \\ \mathrm{H} & -4.640279000 & 2.432946000 & 0.531088000 \\ \mathrm{H} & -5.267639000 & 0.964232000 & 1.281758000 \\ \mathrm{H} & -5.517421000 & 1.244005000 & -0.433321000 \\ \mathrm{C} & -2.306568000 & 1.401357000 & 0.021984000 \\ \mathrm{H} & -2.354093000 & 2.465117000 & 0.211602000 \\ \mathrm{C} & -1.082571000 & 0.813821000 & -0.267427000 \\ \mathrm{~N} & 0.114011000 & 1.563624000 & -0.344855000 \\ \mathrm{C} & 0.055140000 & 3.000330000 & -0.171417000 \\ \mathrm{H} & 1.049854000 & 3.400584000 & -0.331611000 \\ \mathrm{H} & -0.273108000 & 3.282998000 & 0.836258000 \\ \mathrm{H} & 0.242784000 & -2.152916000 & -0.673505000 \\ \mathrm{O} & 0.272523000 & -2.761834000 & 1.760490000 \\ \mathrm{O} & 0.643140000 & -1.783824000 & 2.346450000 \\ \mathrm{H} & -0.637879000 & 3.434514000 & -0.894677000 \\ \mathrm{H} & & \end{array}$

\section{${ }^{1} \mathrm{LFOOH}^{-}$in DMSO}

$\begin{array}{llrl}\mathrm{O} & 2.672240000 & -2.432368000 & -0.770867000 \\ \mathrm{C} & 2.548619000 & -1.264058000 & -0.479566000 \\ \mathrm{~N} & 3.609560000 & -0.401200000 & -0.495369000 \\ \mathrm{H} & 4.520748000 & -0.791337000 & -0.696391000 \\ \mathrm{C} & 3.574642000 & 0.974619000 & -0.229129000 \\ \mathrm{O} & 4.630926000 & 1.579785000 & -0.189532000 \\ \mathrm{~N} & 2.363816000 & 1.577761000 & -0.077075000 \\ \mathrm{C} & 1.268575000 & 0.861235000 & -0.020235000 \\ \mathrm{C} & 1.244494000 & -0.647545000 & 0.011940000 \\ & & & \mathrm{~S} 30\end{array}$




$\begin{array}{lrrr}\mathrm{N} & 0.149445000 & -1.290344000 & -0.518246000 \\ \mathrm{C} & -1.039586000 & -0.635974000 & -0.341397000 \\ \mathrm{C} & -2.254918000 & -1.318656000 & -0.521559000 \\ \mathrm{H} & -2.192517000 & -2.363873000 & -0.805594000 \\ \mathrm{C} & -3.490482000 & -0.723297000 & -0.343137000 \\ \mathrm{C} & -4.751095000 & -1.522307000 & -0.541836000 \\ \mathrm{H} & -5.388171000 & -1.079784000 & -1.312814000 \\ \mathrm{H} & -5.348097000 & -1.564086000 & 0.373725000 \\ \mathrm{H} & -4.522225000 & -2.545471000 & -0.840562000 \\ \mathrm{C} & -3.554007000 & 0.631030000 & 0.033487000 \\ \mathrm{C} & -4.876326000 & 1.316710000 & 0.255074000 \\ \mathrm{H} & -4.732026000 & 2.362383000 & 0.529367000 \\ \mathrm{H} & -5.452143000 & 0.839636000 & 1.053801000 \\ \mathrm{H} & -5.502174000 & 1.291153000 & -0.641933000 \\ \mathrm{C} & -2.367481000 & 1.331763000 & 0.189433000 \\ \mathrm{H} & -2.419870000 & 2.376761000 & 0.464717000 \\ \mathrm{C} & -1.127089000 & 0.725030000 & 0.000439000 \\ \mathrm{~N} & 0.075572000 & 1.466935000 & 0.059224000 \\ \mathrm{C} & 0.003528000 & 2.923966000 & 0.133038000 \\ \mathrm{H} & 1.010316000 & 3.316050000 & 0.060726000 \\ \mathrm{H} & -0.440616000 & 3.228273000 & 1.081465000 \\ \mathrm{H} & 0.366366000 & -2.350669000 & 0.944774000 \\ \mathrm{O} & 0.901946000 & -2.221348000 & 1.771341000 \\ \mathrm{O} & 1.345745000 & -0.880029000 & 1.543252000 \\ \mathrm{H} & -0.605173000 & 3.305757000 & -0.686552000\end{array}$

\section{${ }^{3} \mathrm{LFOOH}^{-}$in DMSO}

$\begin{array}{lrrr}\mathrm{O} & 2.391617000 & -2.284929000 & -1.252950000 \\ \mathrm{C} & 2.407399000 & -1.199750000 & -0.698012000 \\ \mathrm{~N} & 3.485306000 & -0.369972000 & -0.729983000 \\ \mathrm{H} & 4.323261000 & -0.725417000 & -1.169175000 \\ \mathrm{C} & 3.529464000 & 0.986494000 & -0.312777000 \\ \mathrm{O} & 4.634082000 & 1.548055000 & -0.327696000 \\ \mathrm{~N} & 2.368423000 & 1.556945000 & 0.011595000 \\ \mathrm{C} & 1.259622000 & 0.812204000 & 0.059336000 \\ \mathrm{C} & 1.261190000 & -0.652506000 & 0.149695000 \\ \mathrm{~N} & 0.043064000 & -1.364100000 & -0.102201000 \\ \mathrm{C} & -1.077277000 & -0.656462000 & -0.119394000 \\ \mathrm{C} & -2.309118000 & -1.351727000 & -0.293008000 \\ \mathrm{H} & -2.232446000 & -2.424881000 & -0.424531000 \\ \mathrm{C} & -3.530083000 & -0.733338000 & -0.299523000 \\ \mathrm{C} & -4.795270000 & -1.523295000 & -0.493195000 \\ \mathrm{H} & -5.345326000 & -1.189517000 & -1.377203000 \\ \mathrm{H} & -5.470244000 & -1.416177000 & 0.360186000 \\ \mathrm{H} & -4.573312000 & -2.583268000 & -0.615591000 \\ & & & \mathrm{~S} 31\end{array}$




$\begin{array}{lrrr}\mathrm{C} & -3.574173000 & 0.674720000 & -0.112405000 \\ \mathrm{C} & -4.889994000 & 1.396321000 & -0.106133000 \\ \mathrm{H} & -4.753291000 & 2.466170000 & 0.047614000 \\ \mathrm{H} & -5.543956000 & 1.019046000 & 0.685053000 \\ \mathrm{H} & -5.424133000 & 1.253019000 & -1.049604000 \\ \mathrm{C} & -2.396416000 & 1.389089000 & 0.063393000 \\ \mathrm{H} & -2.462875000 & 2.458953000 & 0.201794000 \\ \mathrm{C} & -1.141454000 & 0.774364000 & 0.062790000 \\ \mathrm{~N} & 0.015883000 & 1.473848000 & 0.243537000 \\ \mathrm{C} & -0.014765000 & 2.909937000 & 0.444090000 \\ \mathrm{H} & 1.006881000 & 3.245133000 & 0.582568000 \\ \mathrm{H} & -0.608378000 & 3.159998000 & 1.326531000 \\ \mathrm{H} & 0.836855000 & -2.661769000 & 1.317894000 \\ \mathrm{O} & 1.655201000 & -2.385672000 & 1.768146000 \\ \mathrm{O} & 1.589277000 & -0.949387000 & 1.575462000 \\ \mathrm{H} & -0.442475000 & 3.424367000 & -0.422294000\end{array}$

\section{${ }^{1} \mathrm{LFHOO}^{-}$in DMSO}

$\begin{array}{lrrr}\mathrm{O} & 2.652080000 & -2.266746000 & -1.147674000 \\ \mathrm{C} & 2.562832000 & -1.205674000 & -0.578419000 \\ \mathrm{~N} & 3.649859000 & -0.414207000 & -0.319753000 \\ \mathrm{H} & 4.566745000 & -0.789071000 & -0.523978000 \\ \mathrm{C} & 3.603474000 & 0.926244000 & 0.081172000 \\ \mathrm{O} & 4.645585000 & 1.497815000 & 0.330357000 \\ \mathrm{~N} & 2.386949000 & 1.558421000 & 0.113115000 \\ \mathrm{C} & 1.287729000 & 0.873951000 & -0.010526000 \\ \mathrm{C} & 1.264662000 & -0.640732000 & 0.009640000 \\ \mathrm{~N} & 0.159959000 & -1.176764000 & -0.727664000 \\ \mathrm{C} & -1.069051000 & -0.586682000 & -0.433694000 \\ \mathrm{C} & -2.260921000 & -1.298217000 & -0.505839000 \\ \mathrm{H} & -2.214857000 & -2.352934000 & -0.755113000 \\ \mathrm{C} & -3.492260000 & -0.700121000 & -0.272082000 \\ \mathrm{C} & -4.755511000 & -1.512614000 & -0.360263000 \\ \mathrm{H} & -5.441521000 & -1.103755000 & -1.107077000 \\ \mathrm{H} & -5.292484000 & -1.519093000 & 0.592294000 \\ \mathrm{H} & -4.539037000 & -2.545826000 & -0.631472000 \\ \mathrm{C} & -3.536162000 & 0.663091000 & 0.051475000 \\ \mathrm{C} & -4.846233000 & 1.354620000 & 0.319549000 \\ \mathrm{H} & -4.691333000 & 2.406950000 & 0.558484000 \\ \mathrm{H} & -5.378837000 & 0.895634000 & 1.157128000 \\ \mathrm{H} & -5.512645000 & 1.302212000 & -0.545989000 \\ \mathrm{C} & -2.344977000 & 1.375315000 & 0.126371000 \\ \mathrm{H} & -2.385885000 & 2.424283000 & 0.385769000 \\ \mathrm{C} & -1.116482000 & 0.769017000 & -0.111989000 \\ \mathrm{~N} & 0.099224000 & 1.498231000 & -0.058163000 \\ & & & \mathrm{~S} 32 \\ & & & \end{array}$




$\begin{array}{rrrr}\mathrm{C} & 0.044425000 & 2.956505000 & 0.003050000 \\ \mathrm{H} & 1.046570000 & 3.340127000 & -0.145177000 \\ \mathrm{H} & -0.324437000 & 3.285169000 & 0.976378000 \\ \mathrm{H} & 0.171856000 & -2.169845000 & -0.489942000 \\ \mathrm{O} & 1.035142000 & -2.420228000 & 1.452272000 \\ \mathrm{O} & 1.230148000 & -0.972379000 & 1.377231000 \\ \mathrm{H} & -0.617908000 & 3.327349000 & -0.777874000\end{array}$

\section{The TSs in DMSO}

$\begin{array}{lrrr}\mathrm{O} & 2.667599000 & -2.401288000 & -0.809198000 \\ \mathrm{C} & 2.546028000 & -1.222426000 & -0.501686000 \\ \mathrm{~N} & 3.636613000 & -0.391143000 & -0.359887000 \\ \mathrm{H} & 4.552013000 & -0.801413000 & -0.479108000 \\ \mathrm{C} & 3.594350000 & 0.965094000 & -0.039903000 \\ \mathrm{O} & 4.649936000 & 1.569980000 & 0.095603000 \\ \mathrm{~N} & 2.375390000 & 1.558195000 & 0.086869000 \\ \mathrm{C} & 1.278122000 & 0.836626000 & -0.029309000 \\ \mathrm{C} & 1.286020000 & -0.574283000 & -0.220846000 \\ \mathrm{~N} & 0.140035000 & -1.314390000 & -0.416429000 \\ \mathrm{C} & -1.084746000 & -0.644892000 & -0.268644000 \\ \mathrm{C} & -2.273279000 & -1.358130000 & -0.355941000 \\ \mathrm{H} & -2.211617000 & -2.427861000 & -0.524580000 \\ \mathrm{C} & -3.514027000 & -0.748760000 & -0.233124000 \\ \mathrm{C} & -4.774331000 & -1.565329000 & -0.323527000 \\ \mathrm{H} & -5.412444000 & -1.226953000 & -1.144490000 \\ \mathrm{H} & -5.367725000 & -1.486272000 & 0.591512000 \\ \mathrm{H} & -4.546978000 & -2.618775000 & -0.486683000 \\ \mathrm{C} & -3.563393000 & 0.637057000 & -0.031951000 \\ \mathrm{C} & -4.881499000 & 1.351701000 & 0.098166000 \\ \mathrm{H} & -4.734820000 & 2.419605000 & 0.260183000 \\ \mathrm{H} & -5.466735000 & 0.961369000 & 0.935286000 \\ \mathrm{H} & -5.493215000 & 1.228743000 & -0.799860000 \\ \mathrm{C} & -2.374742000 & 1.353319000 & 0.049910000 \\ \mathrm{H} & -2.429947000 & 2.420034000 & 0.214468000 \\ \mathrm{C} & -1.130224000 & 0.734867000 & -0.056347000 \\ \mathrm{~N} & 0.068948000 & 1.468130000 & 0.048996000 \\ \mathrm{C} & 0.005497000 & 2.905677000 & 0.266224000 \\ \mathrm{H} & 1.020892000 & 3.280336000 & 0.308777000 \\ \mathrm{H} & -0.504710000 & 3.128469000 & 1.205736000 \\ \mathrm{H} & 0.199092000 & -2.153157000 & 0.203418000 \\ \mathrm{O} & 0.721221000 & -2.143037000 & 1.882026000 \\ \mathrm{H} & 1.545329000 & -1.148771000 & 1.820668000 \\ \mathrm{H} & -0.529481000 & 3.391261000 & -0.552058000\end{array}$

${ }^{1}\left(\mathrm{LF}^{-}{ }^{-}{ }^{\cdots} \boldsymbol{O}_{2}\right)$ in water 


\begin{tabular}{|c|c|c|c|}
\hline $\mathrm{O}$ & 2.590640000 & -2.346631000 & -0.664140000 \\
\hline $\mathrm{C}$ & 2.452826000 & -1.129729000 & -0.467257000 \\
\hline $\mathrm{N}$ & 3.536018000 & -0.323152000 & -0.151191000 \\
\hline $\mathrm{H}$ & 4.441521000 & -0.766759000 & -0.097979000 \\
\hline $\mathrm{C}$ & 3.493148000 & 1.051163000 & 0.077477000 \\
\hline $\mathrm{O}$ & 4.549397000 & 1.640549000 & 0.323827000 \\
\hline $\mathrm{N}$ & 2.287026000 & 1.659472000 & 0.022799000 \\
\hline $\mathrm{C}$ & 1.207959000 & 0.929461000 & -0.268726000 \\
\hline $\mathrm{C}$ & 1.230211000 & -0.430412000 & -0.531616000 \\
\hline $\mathrm{N}$ & 0.065444000 & -1.133000000 & -0.912637000 \\
\hline $\mathrm{C}$ & -1.155846000 & -0.558258000 & -0.569207000 \\
\hline $\mathrm{C}$ & -2.327998000 & -1.298710000 & -0.532700000 \\
\hline $\mathrm{H}$ & -2.269787000 & -2.362099000 & -0.741366000 \\
\hline $\mathrm{C}$ & -3.565635000 & -0.721834000 & -0.249818000 \\
\hline $\mathrm{C}$ & -4.808450000 & -1.571160000 & -0.233001000 \\
\hline $\mathrm{H}$ & -5.543400000 & -1.221702000 & -0.963766000 \\
\hline $\mathrm{H}$ & -5.298920000 & -1.544974000 & 0.744246000 \\
\hline $\mathrm{H}$ & -4.575984000 & -2.611191000 & -0.463209000 \\
\hline $\mathrm{C}$ & -3.623312000 & 0.645880000 & 0.015429000 \\
\hline $\mathrm{C}$ & -4.931189000 & 1.324374000 & 0.326665000 \\
\hline $\mathrm{H}$ & -4.785669000 & 2.388994000 & 0.512214000 \\
\hline $\mathrm{H}$ & -5.409260000 & 0.893359000 & 1.211174000 \\
\hline $\mathrm{H}$ & -5.644063000 & 1.223692000 & -0.497028000 \\
\hline $\mathrm{C}$ & -2.440364000 & 1.389683000 & -0.004436000 \\
\hline $\mathrm{H}$ & -2.498054000 & 2.446492000 & 0.217435000 \\
\hline $\mathrm{C}$ & -1.207578000 & 0.819553000 & -0.294381000 \\
\hline $\mathrm{N}$ & -0.018983000 & 1.583209000 & -0.326455000 \\
\hline $\mathrm{C}$ & -0.093916000 & 3.013578000 & -0.108644000 \\
\hline $\mathrm{H}$ & 0.900131000 & 3.425611000 & -0.238752000 \\
\hline $\mathrm{H}$ & -0.442581000 & 3.256299000 & 0.901850000 \\
\hline $\mathrm{H}$ & 0.148320000 & -2.129531000 & -0.754819000 \\
\hline $\mathrm{O}$ & 0.298876000 & -2.516680000 & 1.612602000 \\
\hline $\mathrm{O}$ & 0.827211000 & -1.528372000 & 2.066672000 \\
\hline $\mathrm{H}$ & -0.778150000 & 3.466881000 & -0.828735000 \\
\hline \multicolumn{4}{|c|}{${ }^{3}\left(\mathbf{L F}^{-}{ }_{\text {red }} \cdots \mathbf{O}_{2}\right)$ in water } \\
\hline $\mathrm{O}$ & 2.664827000 & -2.418064000 & -0.376957000 \\
\hline $\mathrm{C}$ & 2.537103000 & -1.190289000 & -0.240981000 \\
\hline $\mathrm{N}$ & 3.616756000 & -0.389824000 & 0.106206000 \\
\hline $\mathrm{H}$ & 4.507857000 & -0.847761000 & 0.231635000 \\
\hline $\mathrm{C}$ & 3.590808000 & 0.994052000 & 0.275235000 \\
\hline $\mathrm{O}$ & 4.646002000 & 1.572189000 & 0.556434000 \\
\hline $\mathrm{N}$ & 2.403130000 & 1.620698000 & 0.129857000 \\
\hline $\mathrm{C}$ & 1.329983000 & 0.894113000 & -0.199271000 \\
\hline $\mathrm{C}$ & 1.339966000 & -0.470066000 & $\begin{array}{l}-0.413794000 \\
\text { S34 }\end{array}$ \\
\hline
\end{tabular}




$\begin{array}{lrrr}\mathrm{N} & 0.174635000 & -1.157997000 & -0.847980000 \\ \mathrm{C} & -1.042960000 & -0.572468000 & -0.499838000 \\ \mathrm{C} & -2.218501000 & -1.303318000 & -0.424689000 \\ \mathrm{H} & -2.171002000 & -2.373274000 & -0.600635000 \\ \mathrm{C} & -3.449901000 & -0.708016000 & -0.147421000 \\ \mathrm{C} & -4.698549000 & -1.547511000 & -0.093461000 \\ \mathrm{H} & -5.436718000 & -1.217305000 & -0.830015000 \\ \mathrm{H} & -5.181810000 & -1.486250000 & 0.885833000 \\ \mathrm{H} & -4.474916000 & -2.596178000 & -0.291215000 \\ \mathrm{C} & -3.495081000 & 0.666771000 & 0.076641000 \\ \mathrm{C} & -4.795063000 & 1.364127000 & 0.380257000 \\ \mathrm{H} & -4.640110000 & 2.432851000 & 0.531714000 \\ \mathrm{H} & -5.267167000 & 0.963952000 & 1.282319000 \\ \mathrm{H} & -5.517298000 & 1.243894000 & -0.432656000 \\ \mathrm{C} & -2.306443000 & 1.401450000 & 0.021962000 \\ \mathrm{H} & -2.353993000 & 2.465189000 & 0.211687000 \\ \mathrm{C} & -1.082518000 & 0.813919000 & -0.267830000 \\ \mathrm{~N} & 0.114162000 & 1.563793000 & -0.345438000 \\ \mathrm{C} & 0.055169000 & 3.000594000 & -0.172280000 \\ \mathrm{H} & 1.049599000 & 3.401146000 & -0.333429000 \\ \mathrm{H} & -0.272375000 & 3.283469000 & 0.835544000 \\ \mathrm{H} & 0.242707000 & -2.152653000 & -0.673901000 \\ \mathrm{O} & 0.272120000 & -2.763168000 & 1.760190000 \\ \mathrm{O} & 0.641021000 & -1.785215000 & 2.347240000 \\ \mathrm{H} & -0.638479000 & 3.434402000 & -0.895115000\end{array}$

\section{${ }^{1} \mathrm{LFOO}_{\mathrm{H}^{-}}$in water}

$\begin{array}{lrrr}\mathrm{O} & 2.672047000 & -2.431944000 & -0.772788000 \\ \mathrm{C} & 2.548628000 & -1.263739000 & -0.480748000 \\ \mathrm{~N} & 3.609603000 & -0.401058000 & -0.495929000 \\ \mathrm{H} & 4.520830000 & -0.791070000 & -0.697131000 \\ \mathrm{C} & 3.574705000 & 0.974457000 & -0.227907000 \\ \mathrm{O} & 4.631115000 & 1.579394000 & -0.187044000 \\ \mathrm{~N} & 2.364031000 & 1.577609000 & -0.075521000 \\ \mathrm{C} & 1.268513000 & 0.861234000 & -0.020106000 \\ \mathrm{C} & 1.244581000 & -0.647456000 & 0.011019000 \\ \mathrm{~N} & 0.149489000 & -1.290398000 & -0.518307000 \\ \mathrm{C} & -1.039718000 & -0.635953000 & -0.341331000 \\ \mathrm{C} & -2.254954000 & -1.318856000 & -0.520940000 \\ \mathrm{H} & -2.192598000 & -2.364212000 & -0.804485000 \\ \mathrm{C} & -3.490540000 & -0.723455000 & -0.342700000 \\ \mathrm{C} & -4.751158000 & -1.522560000 & -0.540823000 \\ \mathrm{H} & -5.388334000 & -1.080361000 & -1.311880000 \\ \mathrm{H} & -5.347999000 & -1.563828000 & 0.374852000 \\ \mathrm{H} & -4.522388000 & -2.545877000 & -0.839090000 \\ & & & \mathrm{~S} 35\end{array}$




$\begin{array}{lrrr}\mathrm{C} & -3.554001000 & 0.631107000 & 0.033182000 \\ \mathrm{C} & -4.876311000 & 1.316906000 & 0.254338000 \\ \mathrm{H} & -4.732058000 & 2.362700000 & 0.528137000 \\ \mathrm{H} & -5.452160000 & 0.840141000 & 1.053203000 \\ \mathrm{H} & -5.502017000 & 1.290845000 & -0.642731000 \\ \mathrm{C} & -2.367512000 & 1.331935000 & 0.188780000 \\ \mathrm{H} & -2.419940000 & 2.377056000 & 0.463526000 \\ \mathrm{C} & -1.127093000 & 0.725073000 & 0.000076000 \\ \mathrm{~N} & 0.075604000 & 1.466958000 & 0.059019000 \\ \mathrm{C} & 0.003467000 & 2.924046000 & 0.133231000 \\ \mathrm{H} & 1.010232000 & 3.316269000 & 0.061694000 \\ \mathrm{H} & -0.441313000 & 3.228017000 & 1.081442000 \\ \mathrm{H} & 0.365913000 & -2.350024000 & 0.943836000 \\ \mathrm{O} & 0.901504000 & -2.221623000 & 1.770695000 \\ \mathrm{O} & 1.347094000 & -0.880851000 & 1.543071000 \\ \mathrm{H} & -0.604718000 & 3.306020000 & -0.686634000\end{array}$

\section{${ }^{3} \mathrm{LFOOH}^{-}$in water}

$\begin{array}{lrrr}\mathrm{O} & 2.390352000 & -2.283284000 & -1.256245000 \\ \mathrm{C} & 2.406756000 & -1.199016000 & -0.699412000 \\ \mathrm{~N} & 3.485095000 & -0.369758000 & -0.730212000 \\ \mathrm{H} & 4.322816000 & -0.724824000 & -1.170195000 \\ \mathrm{C} & 3.529594000 & 0.985976000 & -0.310953000 \\ \mathrm{O} & 4.634557000 & 1.547184000 & -0.324398000 \\ \mathrm{~N} & 2.368734000 & 1.556530000 & 0.013657000 \\ \mathrm{C} & 1.259516000 & 0.812228000 & 0.060124000 \\ \mathrm{C} & 1.261084000 & -0.652573000 & 0.149468000 \\ \mathrm{~N} & 0.042998000 & -1.364183000 & -0.101743000 \\ \mathrm{C} & -1.077401000 & -0.656562000 & -0.118880000 \\ \mathrm{C} & -2.309261000 & -1.351895000 & -0.292256000 \\ \mathrm{H} & -2.232705000 & -2.425103000 & -0.423407000 \\ \mathrm{C} & -3.530162000 & -0.733465000 & -0.299089000 \\ \mathrm{C} & -4.795392000 & -1.523366000 & -0.492483000 \\ \mathrm{H} & -5.345328000 & -1.189828000 & -1.376638000 \\ \mathrm{H} & -5.470358000 & -1.415709000 & 0.360817000 \\ \mathrm{H} & -4.573582000 & -2.583418000 & -0.614403000 \\ \mathrm{C} & -3.574170000 & 0.674742000 & -0.112600000 \\ \mathrm{C} & -4.889891000 & 1.396422000 & -0.106836000 \\ \mathrm{H} & -4.753176000 & 2.466322000 & 0.046444000 \\ \mathrm{H} & -5.543953000 & 1.019457000 & 0.684403000 \\ \mathrm{H} & -5.423867000 & 1.252638000 & -1.050316000 \\ \mathrm{C} & -2.396348000 & 1.389094000 & 0.063076000 \\ \mathrm{H} & -2.462814000 & 2.459005000 & 0.201048000 \\ \mathrm{C} & -1.141532000 & 0.774265000 & 0.062964000 \\ \mathrm{~N} & 0.015932000 & 1.473863000 & 0.243931000 \\ & & & \mathrm{~S} 36 \\ & & & \end{array}$




$\begin{array}{rrrr}\mathrm{C} & -0.014938000 & 2.910146000 & 0.443548000 \\ \mathrm{H} & 1.006511000 & 3.245607000 & 0.582692000 \\ \mathrm{H} & -0.609215000 & 3.160694000 & 1.325366000 \\ \mathrm{H} & 0.837293000 & -2.662162000 & 1.318324000 \\ \mathrm{O} & 1.656772000 & -2.386425000 & 1.766757000 \\ \mathrm{O} & 1.590672000 & -0.950098000 & 1.574911000 \\ \mathrm{H} & -0.442052000 & 3.423990000 & -0.423455000\end{array}$

\section{${ }^{1} \mathrm{LF}_{\mathrm{HOO}} \mathrm{OO}^{-}$in water}

$\begin{array}{lrrr}\mathrm{O} & 2.651803000 & -2.267090000 & -1.146717000 \\ \mathrm{C} & 2.562752000 & -1.205856000 & -0.577602000 \\ \mathrm{~N} & 3.649738000 & -0.414633000 & -0.318772000 \\ \mathrm{H} & 4.566640000 & -0.789380000 & -0.523275000 \\ \mathrm{C} & 3.603303000 & 0.925934000 & 0.082108000 \\ \mathrm{O} & 4.645509000 & 1.497204000 & 0.331789000 \\ \mathrm{~N} & 2.387036000 & 1.558333000 & 0.113672000 \\ \mathrm{C} & 1.287616000 & 0.874066000 & -0.010434000 \\ \mathrm{C} & 1.264427000 & -0.640683000 & 0.009897000 \\ \mathrm{~N} & 0.160179000 & -1.176027000 & -0.728428000 \\ \mathrm{C} & -1.069117000 & -0.586497000 & -0.434184000 \\ \mathrm{C} & -2.260888000 & -1.298159000 & -0.506370000 \\ \mathrm{H} & -2.214925000 & -2.352774000 & -0.756128000 \\ \mathrm{C} & -3.492207000 & -0.700145000 & -0.272152000 \\ \mathrm{C} & -4.755469000 & -1.512569000 & -0.360474000 \\ \mathrm{H} & -5.441537000 & -1.103309000 & -1.106995000 \\ \mathrm{H} & -5.292308000 & -1.519346000 & 0.592146000 \\ \mathrm{H} & -4.539115000 & -2.545676000 & -0.632157000 \\ \mathrm{C} & -3.536068000 & 0.662974000 & 0.051981000 \\ \mathrm{C} & -4.846105000 & 1.354323000 & 0.320598000 \\ \mathrm{H} & -4.691223000 & 2.406542000 & 0.559973000 \\ \mathrm{H} & -5.378529000 & 0.894877000 & 1.158025000 \\ \mathrm{H} & -5.512609000 & 1.302216000 & -0.544870000 \\ \mathrm{C} & -2.344926000 & 1.375262000 & 0.126836000 \\ \mathrm{H} & -2.385819000 & 2.424149000 & 0.386558000 \\ \mathrm{C} & -1.116492000 & 0.769076000 & -0.112124000 \\ \mathrm{~N} & 0.099294000 & 1.498320000 & -0.058541000 \\ \mathrm{C} & 0.044414000 & 2.956727000 & 0.001712000 \\ \mathrm{H} & 1.046240000 & 3.340474000 & -0.148246000 \\ \mathrm{H} & -0.323178000 & 3.286056000 & 0.975279000 \\ \mathrm{H} & 0.171891000 & -2.169715000 & -0.494386000 \\ \mathrm{O} & 1.036141000 & -2.419714000 & 1.453876000 \\ \mathrm{H} & 1.229257000 & -0.971706000 & 1.377594000 \\ \mathrm{H} & -0.619027000 & 3.326794000 & -0.778594000\end{array}$

The TSs in water 


$\begin{array}{lrrr}\mathrm{O} & 2.667421000 & -2.401682000 & -0.807889000 \\ \mathrm{C} & 2.545937000 & -1.222472000 & -0.501148000 \\ \mathrm{~N} & 3.636673000 & -0.391473000 & -0.359042000 \\ \mathrm{H} & 4.552055000 & -0.801916000 & -0.477940000 \\ \mathrm{C} & 3.594401000 & 0.964853000 & -0.039554000 \\ \mathrm{O} & 4.650152000 & 1.569631000 & 0.096219000 \\ \mathrm{~N} & 2.375651000 & 1.558146000 & 0.086786000 \\ \mathrm{C} & 1.278115000 & 0.836729000 & -0.029873000 \\ \mathrm{C} & 1.286023000 & -0.573992000 & -0.221824000 \\ \mathrm{~N} & 0.140012000 & -1.313992000 & -0.417387000 \\ \mathrm{C} & -1.084851000 & -0.644711000 & -0.269115000 \\ \mathrm{C} & -2.273369000 & -1.358029000 & -0.356118000 \\ \mathrm{H} & -2.211828000 & -2.427750000 & -0.524889000 \\ \mathrm{C} & -3.514075000 & -0.748630000 & -0.232977000 \\ \mathrm{C} & -4.774407000 & -1.565156000 & -0.323128000 \\ \mathrm{H} & -5.413222000 & -1.225888000 & -1.143149000 \\ \mathrm{H} & -5.366977000 & -1.487020000 & 0.592516000 \\ \mathrm{H} & -4.547188000 & -2.618426000 & -0.487575000 \\ \mathrm{C} & -3.563357000 & 0.637215000 & -0.031679000 \\ \mathrm{C} & -4.881397000 & 1.351884000 & 0.098766000 \\ \mathrm{H} & -4.734650000 & 2.419634000 & 0.261687000 \\ \mathrm{H} & -5.466827000 & 0.960864000 & 0.935404000 \\ \mathrm{H} & -5.492874000 & 1.229699000 & -0.799520000 \\ \mathrm{C} & -2.374689000 & 1.353478000 & 0.049928000 \\ \mathrm{H} & -2.429892000 & 2.420182000 & 0.214524000 \\ \mathrm{C} & -1.130211000 & 0.735021000 & -0.056729000 \\ \mathrm{~N} & 0.069080000 & 1.468314000 & 0.048329000 \\ \mathrm{C} & 0.005547000 & 2.905957000 & 0.265366000 \\ \mathrm{H} & 1.020858000 & 3.280828000 & 0.307558000 \\ \mathrm{H} & -0.504392000 & 3.128843000 & 1.204981000 \\ \mathrm{H} & 0.199002000 & -2.153964000 & 0.200421000 \\ \mathrm{O} & 0.721290000 & -2.144142000 & 1.882070000 \\ \mathrm{H} & 1.544913000 & -1.149738000 & 1.822080000 \\ & -0.529751000 & 3.391280000 & -0.552825000\end{array}$

The XYZ coordinates of stationary points computed at (U)CAM-B3LYP/6-311+G** level ${ }^{1}\left(\mathrm{LF}^{-}{ }^{-}{ }^{\cdots}{ }^{\cdots} \mathbf{O}_{2}\right)$ in the gas phase

$\begin{array}{llrl}8 & 2.679722000 & -2.429156000 & -0.574502000 \\ 6 & 2.557218000 & -1.213726000 & -0.368627000 \\ 7 & 3.650575000 & -0.403376000 & -0.105442000 \\ 1 & 4.556469000 & -0.846716000 & -0.084297000 \\ 6 & 3.625600000 & 0.978340000 & 0.120106000 \\ 8 & 4.691052000 & 1.557407000 & 0.319339000 \\ & & & \mathrm{~S} 38\end{array}$




$\begin{array}{rrrr}7 & 2.411578000 & 1.587620000 & 0.103509000 \\ 6 & 1.332150000 & 0.855463000 & -0.134659000 \\ 6 & 1.332055000 & -0.509594000 & -0.371608000 \\ 7 & 0.152094000 & -1.204087000 & -0.690131000 \\ 6 & -1.061271000 & -0.610199000 & -0.413810000 \\ 6 & -2.250858000 & -1.324874000 & -0.410532000 \\ 1 & -2.205000000 & -2.393165000 & -0.597321000 \\ 6 & -3.486835000 & -0.720788000 & -0.180208000 \\ 6 & -4.744162000 & -1.549867000 & -0.193344000 \\ 1 & -5.450209000 & -1.204636000 & -0.955663000 \\ 1 & -5.267714000 & -1.505669000 & 0.767087000 \\ 1 & -4.518284000 & -2.597144000 & -0.399695000 \\ 6 & -3.529424000 & 0.647566000 & 0.065599000 \\ 6 & -4.833755000 & 1.354378000 & 0.326536000 \\ 1 & -4.670965000 & 2.419357000 & 0.500997000 \\ 1 & -5.348134000 & 0.950821000 & 1.205192000 \\ 1 & -5.526762000 & 1.259941000 & -0.516367000 \\ 6 & -2.330539000 & 1.369824000 & 0.077121000 \\ 1 & -2.375198000 & 2.430882000 & 0.281862000 \\ 6 & -1.097311000 & 0.777825000 & -0.159080000 \\ 7 & 0.097072000 & 1.519483000 & -0.166535000 \\ 6 & 0.048418000 & 2.941848000 & 0.081247000 \\ 1 & 1.064575000 & 3.319743000 & 0.021694000 \\ 1 & -0.351288000 & 3.164962000 & 1.078777000 \\ 1 & 0.235322000 & -2.208024000 & -0.604226000 \\ 8 & 0.331970000 & -2.341447000 & 1.952552000 \\ 8 & 1.006293000 & -1.371560000 & 2.235236000 \\ 1 & -0.581497000 & 3.435820000 & -0.664958000\end{array}$

\section{${ }^{1} \mathrm{LFOOH}^{-}$in the gas phase}

$\begin{array}{lrrr}8 & 2.685015000 & -2.441308000 & -0.745191000 \\ 6 & 2.545394000 & -1.277484000 & -0.459217000 \\ 7 & 3.604075000 & -0.399160000 & -0.500376000 \\ 1 & 4.512929000 & -0.789898000 & -0.704401000 \\ 6 & 3.568161000 & 0.985823000 & -0.293084000 \\ 8 & 4.619314000 & 1.599403000 & -0.308530000 \\ 7 & 2.348532000 & 1.581364000 & -0.140104000 \\ 6 & 1.270900000 & 0.854679000 & -0.019065000 \\ 6 & 1.241714000 & -0.658729000 & 0.052498000 \\ 7 & 0.137630000 & -1.295354000 & -0.485473000 \\ 6 & -1.039194000 & -0.641369000 & -0.324513000 \\ 6 & -2.259666000 & -1.314071000 & -0.532172000 \\ 1 & -2.193130000 & -2.355436000 & -0.828638000 \\ 6 & -3.493113000 & -0.715424000 & -0.358545000 \\ 6 & -4.754420000 & -1.509826000 & -0.579547000\end{array}$




$\begin{array}{lrrr}1 & -5.385698000 & -1.059905000 & -1.352725000 \\ 1 & -5.360648000 & -1.567789000 & 0.330409000 \\ 1 & -4.520452000 & -2.529081000 & -0.889686000 \\ 6 & -3.560392000 & 0.632972000 & 0.032242000 \\ 6 & -4.882763000 & 1.318798000 & 0.254531000 \\ 1 & -4.734912000 & 2.363565000 & 0.535419000 \\ 1 & -5.464009000 & 0.843786000 & 1.052623000 \\ 1 & -5.511101000 & 1.305150000 & -0.642884000 \\ 6 & -2.370866000 & 1.328656000 & 0.204824000 \\ 1 & -2.423068000 & 2.372321000 & 0.488852000 \\ 6 & -1.131538000 & 0.723399000 & 0.021970000 \\ 7 & 0.071098000 & 1.461327000 & 0.082484000 \\ 6 & 0.007597000 & 2.912667000 & 0.154720000 \\ 1 & 1.020233000 & 3.296786000 & 0.096117000 \\ 1 & -0.449270000 & 3.220079000 & 1.098037000 \\ 1 & 0.412996000 & -2.406867000 & 1.039859000 \\ 8 & 0.974182000 & -2.212959000 & 1.829682000 \\ 8 & 1.325110000 & -0.852067000 & 1.557212000 \\ 1 & -0.588095000 & 3.302454000 & -0.673322000\end{array}$

\section{The TSs in the gas phase}

$\begin{array}{lrrr}8 & 2.667598000 & -2.382537000 & -0.908895000 \\ 6 & 2.545489000 & -1.229845000 & -0.535708000 \\ 7 & 3.631126000 & -0.382295000 & -0.424180000 \\ 1 & 4.544403000 & -0.783197000 & -0.577585000 \\ 6 & 3.594077000 & 0.971122000 & -0.084652000 \\ 8 & 4.645120000 & 1.582238000 & 0.016604000 \\ 7 & 2.364975000 & 1.553057000 & 0.071143000 \\ 6 & 1.284187000 & 0.817627000 & 0.006407000 \\ 6 & 1.290670000 & -0.608016000 & -0.127385000 \\ 7 & 0.132632000 & -1.333410000 & -0.344929000 \\ 6 & -1.083481000 & -0.658479000 & -0.229694000 \\ 6 & -2.276433000 & -1.361950000 & -0.330578000 \\ 1 & -2.214713000 & -2.434142000 & -0.482928000 \\ 6 & -3.517387000 & -0.745975000 & -0.236392000 \\ 6 & -4.778456000 & -1.562661000 & -0.335729000 \\ 1 & -5.407662000 & -1.238393000 & -1.170763000 \\ 1 & -5.385274000 & -1.478935000 & 0.571330000 \\ 1 & -4.546451000 & -2.618094000 & -0.483307000 \\ 6 & -3.567037000 & 0.638527000 & -0.052855000 \\ 6 & -4.884539000 & 1.361279000 & 0.047698000 \\ 1 & -4.732418000 & 2.430899000 & 0.200904000 \\ 1 & -5.487969000 & 0.988819000 & 0.881545000 \\ 1 & -5.485999000 & 1.237786000 & -0.858686000 \\ 6 & -2.374520000 & 1.349632000 & 0.043181000 \\ & & & \mathrm{~S} 40\end{array}$




$\begin{array}{rrrr}1 & -2.425479000 & 2.418559000 & 0.198859000 \\ 6 & -1.131250000 & 0.725875000 & -0.031250000 \\ 7 & 0.065728000 & 1.450022000 & 0.092514000 \\ 6 & 0.015101000 & 2.879469000 & 0.328075000 \\ 1 & 1.037061000 & 3.234681000 & 0.411857000 \\ 1 & -0.526263000 & 3.093234000 & 1.253950000 \\ 1 & 0.178326000 & -2.162745000 & 0.294252000 \\ 8 & 0.732974000 & -2.071514000 & 1.958022000 \\ 8 & 1.571373000 & -1.088449000 & 1.774554000 \\ 1 & -0.482335000 & 3.391389000 & -0.501105000\end{array}$

The XYZ coordinates of stationary points computed at (U)CAM-B3LYP/6-311++G** level ${ }^{1}\left(\mathrm{LF}^{-}{ }^{-}{ }^{\cdots} \cdots \mathrm{O}_{2}\right)$ in the gas phase

\begin{tabular}{lrrr}
8 & 2.680051000 & -2.429242000 & -0.576012000 \\
6 & 2.557526000 & -1.213756000 & -0.370425000 \\
7 & 3.651120000 & -0.402992000 & -0.109535000 \\
1 & 4.557058000 & -0.846230000 & -0.088805000 \\
6 & 3.626036000 & 0.978564000 & 0.116901000 \\
8 & 4.691536000 & 1.557833000 & 0.315281000 \\
7 & 2.411847000 & 1.587554000 & 0.101887000 \\
6 & 1.332174000 & 0.855067000 & -0.134307000 \\
6 & 1.332089000 & -0.510044000 & -0.371119000 \\
7 & 0.151814000 & -1.205247000 & -0.686347000 \\
6 & -1.061502000 & -0.610793000 & -0.411588000 \\
6 & -2.251281000 & -1.325191000 & -0.409165000 \\
1 & -2.205515000 & -2.393472000 & -0.595971000 \\
6 & -3.487356000 & -0.720773000 & -0.180238000 \\
6 & -4.744814000 & -1.549579000 & -0.194229000 \\
1 & -5.451120000 & -1.202843000 & -0.955627000 \\
1 & -5.267981000 & -1.506837000 & 0.766500000 \\
1 & -4.519176000 & -2.596549000 & -0.402447000 \\
6 & -3.529902000 & 0.647710000 & 0.064872000 \\
6 & -4.834343000 & 1.354887000 & 0.324068000 \\
1 & -4.671502000 & 2.419915000 & 0.498228000 \\
1 & -5.349858000 & 0.951738000 & 1.202262000 \\
1 & -5.526443000 & 1.260186000 & -0.519577000 \\
6 & -2.330844000 & 1.369693000 & 0.077257000 \\
1 & -2.375467000 & 2.430890000 & 0.281180000 \\
6 & -1.097494000 & 0.777362000 & -0.157487000 \\
7 & 0.096958000 & 1.518885000 & -0.163778000 \\
6 & 0.048271000 & 2.941105000 & 0.084574000 \\
1 & 1.064793000 & 3.318501000 & 0.027619000 \\
1 & -0.353799000 & 3.163823000 & 1.081247000 \\
& & & $\mathrm{~S} 41$ \\
\hline & & & \\
6 & & &
\end{tabular}




$\begin{array}{rrrr}1 & 0.235140000 & -2.209057000 & -0.599187000 \\ 8 & 0.334119000 & -2.339308000 & 1.955233000 \\ 8 & 1.010404000 & -1.370566000 & 2.237373000 \\ 1 & -0.579584000 & 3.436016000 & -0.662789000\end{array}$

\section{${ }^{1} \mathrm{LFOO}_{\mathrm{H}^{-}}$in the gas phase}$$
8
$$

6

$$
7
$$$$
1
$$$$
6
$$$$
8
$$$$
7
$$$$
6
$$$$
6
$$

2.684811000

2.545315000

3.603948000

4.512799000

3.568158000

4.619338000

2.348521000

1.270844000

1.241934000

0.137522000

$-1.039294000$

$-2.259832000$

$-2.193367000$

$-3.493272000$

$-4.754565000$

$-5.385599000$

$-5.361093000$

$-4.520519000$

$-3.560540000$

$-4.882870000$

$-4.734897000$

$-5.464690000$

$-5.510750000$

$-2.370948000$

$-2.423100000$

$-1.131627000$

0.071013000

0.007473000

1.020230000

$-0.450428000$

0.413448000

0.974777000

1.326985000

$-0.587184000$
$-2.440947000$

$-1.277247000$

$-0.398881000$

$-0.789636000$

0.985852000

1.599436000

1.581304000

0.854581000

$-0.658818000$

$-1.295801000$

$-0.641723000$

$-1.314321000$

$-2.355775000$

$-0.715433000$

$-1.509869000$

$-1.060521000$

$-1.566944000$

$-2.529408000$

0.633034000

1.319108000

2.363841000

0.844182000

1.305541000

1.328615000

2.372440000

0.723219000

1.461169000

2.912463000

3.296505000

3.219609000

$-2.406296000$

$-2.213070000$

$-0.852446000$

3.302737000
$-0.746995000$

$-0.460331000$

$-0.501700000$

$-0.705643000$

$-0.292813000$

$-0.307349000$

$-0.139413000$

$-0.019002000$

0.052538000

$-0.484408000$

$-0.323629000$

$-0.531380000$

$-0.827544000$

$-0.358419000$

$-0.579174000$

$-1.352905000$

0.330644000

$-0.888355000$

0.032066000

0.253675000

0.534667000

1.051412000

$-0.644081000$

0.204662000

0.488043000

0.022196000

0.082542000

0.155251000

0.097845000

1.098134000

1.039752000

1.829764000

1.557266000

$-0.673311000$

The TSs in the gas phase

$\begin{array}{llll}8 & 2.667148000 & -2.382129000 & -0.911599000 \\ 6 & 2.545142000 & -1.229713000 & -0.537420000 \\ 7 & 3.630734000 & -0.382012000 & -0.426860000\end{array}$




$\begin{array}{lrrr}1 & 4.543853000 & -0.782792000 & -0.581471000 \\ 6 & 3.593934000 & 0.971164000 & -0.086249000 \\ 8 & 4.645045000 & 1.582237000 & 0.014765000 \\ 7 & 2.364930000 & 1.552935000 & 0.070651000 \\ 6 & 1.284172000 & 0.817321000 & 0.006679000 \\ 6 & 1.290702000 & -0.608354000 & -0.127039000 \\ 7 & 0.132511000 & -1.334138000 & -0.342429000 \\ 6 & -1.083556000 & -0.658800000 & -0.228491000 \\ 6 & -2.276532000 & -1.362204000 & -0.329515000 \\ 1 & -2.214831000 & -2.434425000 & -0.481610000 \\ 6 & -3.517482000 & -0.746038000 & -0.236465000 \\ 6 & -4.778530000 & -1.562684000 & -0.335955000 \\ 1 & -5.408179000 & -1.237634000 & -1.170352000 \\ 1 & -5.384934000 & -1.479789000 & 0.571476000 \\ 1 & -4.546434000 & -2.617954000 & -0.484631000 \\ 6 & -3.567067000 & 0.638553000 & -0.053546000 \\ 6 & -4.884519000 & 1.361524000 & 0.045714000 \\ 1 & -4.732322000 & 2.431053000 & 0.199532000 \\ 1 & -5.488994000 & 0.988770000 & 0.878679000 \\ 1 & -5.484974000 & 1.238473000 & -0.861411000 \\ 6 & -2.374510000 & 1.349556000 & 0.042851000 \\ 1 & -2.425396000 & 2.418566000 & 0.197928000 \\ 6 & -1.131273000 & 0.725572000 & -0.030328000 \\ 7 & 0.065716000 & 1.449550000 & 0.094123000 \\ 6 & 0.015099000 & 2.878880000 & 0.330274000 \\ 1 & 1.037071000 & 3.233806000 & 0.415452000 \\ 1 & -0.527548000 & 3.092390000 & 1.255434000 \\ 1 & 0.178305000 & -2.161791000 & 0.299189000 \\ 8 & 0.733128000 & -2.069289000 & 1.960781000 \\ 8 & 1.573720000 & -1.088495000 & 1.775456000 \\ 1 & -0.480957000 & 3.391468000 & -0.499340000\end{array}$

The XYZ coordinates of stationary points computed at (U)wB97XD/6-311G** level ${ }^{1}\left(\mathrm{LF}^{-}{ }^{-}{ }^{\cdots}{ }^{\cdots} \mathrm{O}_{2}\right)$ in the gas phase

$\begin{array}{lrrr}8 & 2.681477000 & -2.427306000 & -0.589362000 \\ 6 & 2.560505000 & -1.219500000 & -0.383800000 \\ 7 & 3.650244000 & -0.402112000 & -0.127291000 \\ 1 & 4.555945000 & -0.842976000 & -0.107111000 \\ 6 & 3.629266000 & 0.981695000 & 0.101948000 \\ 8 & 4.689598000 & 1.560524000 & 0.292423000 \\ 7 & 2.410001000 & 1.592710000 & 0.094403000 \\ 6 & 1.331353000 & 0.859965000 & -0.127540000 \\ 6 & 1.326826000 & -0.509470000 & -0.371753000\end{array}$


$-0.667066000$

$-0.398880000$

$-0.393451000$

$-2.188980000$

$-2.395617000$

$-0.561563000$

$-0.720575000$

$-0.176013000$

$-4.740216000$

$-1.549748000$

$-0.173869000$

$-5.450481000$

$-1.209335000$

$-0.936072000$

$-5.256734000$

$-1.491571000$

0.790957000

$-4.518115000$

$-2.600873000$

$-0.369206000$

$-3.528660000$

0.654152000

0.045624000

$-4.839472000$

1.356312000

0.290783000

$-4.687153000$

2.426729000

0.446392000

$-5.349469000$

0.961004000

1.177008000

$-5.530320000$

1.237458000

$-0.552121000$

$-2.333346000$

1.380614000

0.055444000

$-2.382202000$

2.445350000

0.247397000

$-1.094611000$

0.784728000

$-0.156620000$

0.096949000

1.521988000

$-0.137890000$

0.051576000

2.941339000

0.119483000

1.075990000

3.305134000

0.102646000

$-0.386924000$

3.155614000

1.103392000

0.232090000

$-2.197168000$

$-0.430171000$

0.325150000

$-2.378034000$

1.826285000

0.981694000

$-1.391731000$

2.158602000

$-0.542798000$

3.451599000

$-0.647160000$

\section{${ }^{1} \mathrm{LFOOH}^{-}$in the gas phase}

2.688925000

$-2.450958000$

$-0.705683000$

$-0.440192000$

2.545580000

$-1.283303000$

$-0.496313000$

3.601112000

$-0.399545000$

$-0.685484000$

3.571423000

$-0.793358000$

$-0.288704000$

4.620988000

0.987487000

$-0.295732000$

2.348057000

1.597760000

$-0.148422000$

1.273496000

1.586892000

$-0.020167000$

1.240214000

0.857123000

0.063379000

0.141903000

$-0.658212000$

$-0.505540000$

$-1.032068000$

$-1.292513000$

$-0.336595000$

$-2.255510000$

$-0.640361000$

$-0.547247000$

$-2.185834000$

$-0.853650000$

$-3.489661000$

$-2.350265000$

$-0.364293000$

$-4.754127000$

$-0.713945000$

$-0.591012000$

$-5.380201000$

$-1.503813000$

$-1.366970000$

$-5.363782000$

$-1.048126000$

0.318452000

$-4.525232000$

$-1.557085000$

$-0.900829000$ 


$\begin{array}{lrrr}6 & -3.558275000 & 0.630192000 & 0.043094000 \\ 6 & -4.883516000 & 1.308252000 & 0.277515000 \\ 1 & -4.740960000 & 2.356649000 & 0.552163000 \\ 1 & -5.451769000 & 0.830292000 & 1.085052000 \\ 1 & -5.521684000 & 1.284821000 & -0.614369000 \\ 6 & -2.368103000 & 1.326423000 & 0.219279000 \\ 1 & -2.420901000 & 2.368482000 & 0.515567000 \\ 6 & -1.128749000 & 0.724283000 & 0.024728000 \\ 7 & 0.071896000 & 1.463993000 & 0.080297000 \\ 6 & 0.009533000 & 2.914014000 & 0.134237000 \\ 1 & 1.023000000 & 3.294243000 & 0.044627000 \\ 1 & -0.424354000 & 3.236689000 & 1.085066000 \\ 1 & 0.410226000 & -2.400648000 & 1.028895000 \\ 8 & 0.966184000 & -2.208244000 & 1.816498000 \\ 8 & 1.292345000 & -0.845818000 & 1.551693000 \\ 1 & -0.607869000 & 3.291424000 & -0.685333000\end{array}$

\section{The TSs in the gas phase}

\begin{tabular}{lrrr}
8 & 2.668175000 & -2.391756000 & -0.929506000 \\
6 & 2.540377000 & -1.240157000 & -0.563668000 \\
7 & 3.622162000 & -0.381533000 & -0.465895000 \\
1 & 4.534374000 & -0.781483000 & -0.618425000 \\
6 & 3.592294000 & 0.969637000 & -0.108895000 \\
8 & 4.640949000 & 1.577286000 & -0.003903000 \\
7 & 2.360632000 & 1.552438000 & 0.058729000 \\
6 & 1.283770000 & 0.811600000 & 0.003257000 \\
6 & 1.285703000 & -0.614012000 & -0.149085000 \\
7 & 0.130420000 & -1.356116000 & -0.314651000 \\
6 & -1.080379000 & -0.664801000 & -0.217869000 \\
6 & -2.275119000 & -1.365959000 & -0.330663000 \\
1 & -2.207150000 & -2.438446000 & -0.484129000 \\
6 & -3.517319000 & -0.748904000 & -0.244128000 \\
6 & -4.781952000 & -1.561560000 & -0.346540000 \\
1 & -5.422418000 & -1.213892000 & -1.164946000 \\
1 & -5.374880000 & -1.496133000 & 0.572805000 \\
1 & -4.555965000 & -2.615067000 & -0.522206000 \\
6 & -3.566512000 & 0.636529000 & -0.060244000 \\
6 & -4.886730000 & 1.358041000 & 0.027404000 \\
1 & -4.739292000 & 2.426634000 & 0.198525000 \\
1 & -5.504899000 & 0.970564000 & 0.844827000 \\
1 & -5.469408000 & 1.244937000 & -0.893990000 \\
6 & -2.374132000 & 1.348337000 & 0.044439000 \\
1 & -2.426305000 & 2.418930000 & 0.199546000 \\
6 & -1.129819000 & 0.722055000 & -0.019528000 \\
7 & 0.064287000 & 1.442557000 & 0.111265000 \\
& & & $\mathrm{~S} 45$ \\
\hline & & &
\end{tabular}




$\begin{array}{rrrr}6 & 0.019735000 & 2.868107000 & 0.358686000 \\ 1 & 1.046819000 & 3.211556000 & 0.449824000 \\ 1 & -0.525970000 & 3.076914000 & 1.284701000 \\ 1 & 0.185685000 & -2.093798000 & 0.460001000 \\ 8 & 0.717515000 & -2.005177000 & 1.967107000 \\ 8 & 1.607523000 & -1.075840000 & 1.761699000 \\ 1 & -0.468996000 & 3.392090000 & -0.470272000\end{array}$

The XYZ coordinates of the optimized ISC point computed at CASSCF/6-31G* level

\section{$\mathbf{T}_{1} / \mathbf{S}_{0}$ intersection}

$\begin{array}{lccc}\mathrm{O} & 2.56178822 & -2.35060580 & -1.00576765 \\ \mathrm{C} & 2.40582399 & -1.19480262 & -0.68008019 \\ \mathrm{~N} & 3.47792309 & -0.31116966 & -0.69540069 \\ \mathrm{H} & 4.37244377 & -0.68924737 & -0.91443524 \\ \mathrm{C} & 3.44441697 & 1.02982272 & -0.38526190 \\ \mathrm{O} & 4.46677273 & 1.66700840 & -0.39947885 \\ \mathrm{~N} & 2.22048980 & 1.59148888 & -0.10703753 \\ \mathrm{C} & 1.17280002 & 0.82241581 & -0.04537509 \\ \mathrm{C} & 1.18700421 & -0.58992769 & -0.20629548 \\ \mathrm{~N} & 0.01573055 & -1.35537423 & -0.22506667 \\ \mathrm{C} & -1.20117485 & -0.66357436 & -0.19050449 \\ \mathrm{C} & -2.38688239 & -1.35364399 & -0.36433326 \\ \mathrm{H} & -2.32186570 & -2.41614567 & -0.52396739 \\ \mathrm{C} & -3.62842082 & -0.73221364 & -0.33743707 \\ \mathrm{C} & -4.88889519 & -1.54816229 & -0.51479594 \\ \mathrm{H} & -5.45898862 & -1.22969595 & -1.38459845 \\ \mathrm{H} & -5.54878385 & -1.46493950 & 0.34577072 \\ \mathrm{H} & -4.65300747 & -2.59818460 & -0.64505302 \\ \mathrm{C} & -3.67215852 & 0.64697114 & -0.16038538 \\ \mathrm{C} & -4.98699208 & 1.39430583 & -0.13915921 \\ \mathrm{H} & -4.82796840 & 2.45606605 & 0.01288514 \\ \mathrm{H} & -5.63780293 & 1.04324733 & 0.65827592 \\ \mathrm{H} & -5.53462554 & 1.27551241 & -1.07128927 \\ \mathrm{C} & -2.48124451 & 1.34801528 & -0.00325708 \\ \mathrm{H} & -2.53441812 & 2.41090439 & 0.12997723 \\ \mathrm{C} & -1.24180853 & 0.71910223 & 0.00287970 \\ \mathrm{~N} & -0.05027793 & 1.42865437 & 0.19646054 \\ \mathrm{C} & -0.10212104 & 2.83508811 & 0.52334184 \\ \mathrm{H} & 0.89134142 & 3.16628286 & 0.76405221 \\ \mathrm{H} & -0.75409270 & 2.99241852 & 1.37646655 \\ \mathrm{H} & 0.05898442 & -2.07744080 & 0.48243350 \\ \mathrm{O} & 0.79170695 & -1.75314341 & 2.28482683 \\ \mathrm{O} & 1.67456628 & -0.95961704 & 1.88862207\end{array}$


$\begin{array}{llll}\mathrm{H} & -0.47249221 & 3.42953022 & -0.30956540\end{array}$ 